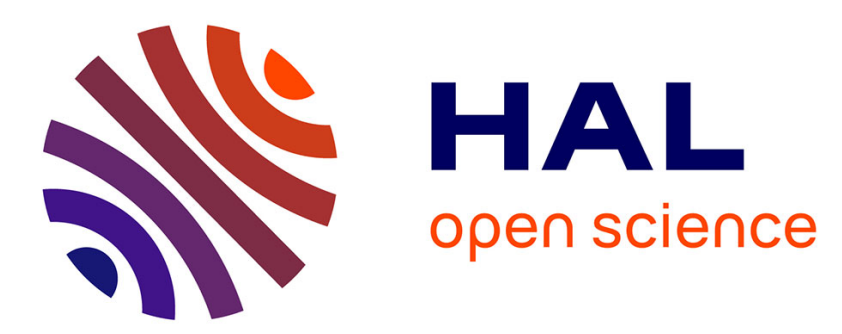

\title{
Fresnel Line-of-Sight Probability with Applications in Airborne Platform-Assisted Communications
}

\author{
Yassine Hmamouche, Mustapha Benjillali, Samir Saoudi
}

\section{To cite this version:}

Yassine Hmamouche, Mustapha Benjillali, Samir Saoudi. Fresnel Line-of-Sight Probability with Applications in Airborne Platform-Assisted Communications. IEEE Transactions on Vehicular Technology, 2022, 10.1109/TVT.2022.3151461 . hal-03566664

\section{HAL Id: hal-03566664 https://hal-imt.archives-ouvertes.fr/hal-03566664}

Submitted on 11 Feb 2022

HAL is a multi-disciplinary open access archive for the deposit and dissemination of scientific research documents, whether they are published or not. The documents may come from teaching and research institutions in France or abroad, or from public or private research centers.
L'archive ouverte pluridisciplinaire HAL, est destinée au dépôt et à la diffusion de documents scientifiques de niveau recherche, publiés ou non, émanant des établissements d'enseignement et de recherche français ou étrangers, des laboratoires publics ou privés. 


\title{
Fresnel Line-of-Sight Probability with Applications in Airborne Platform-Assisted Communications
}

\author{
Yassine Hmamouche, Member, IEEE, Mustapha Benjillali, Senior Member, IEEE, \\ and Samir Saoudi, Senior Member, IEEE
}

\begin{abstract}
The ecosystem of airborne platforms is maturing rapidly and becoming essential to meeting the communication requirements of modern wireless networks. In such a context, the speed, range, and quality of service are highly dependent on a timely access to the right amount and type of affordable spectrum. In this way, evaluating the statistical properties of the air-to-ground (AtG) channel in different built-up propagation environments with regard to the operating frequency is crucial for performance analysis of airborne platform-assisted communications. In this paper, we construct a framework for the line-of-sight (LoS) probability based on the intrusion ratio of obstacles within the first Fresnel zone, yielding an analytical expression for the LoS probability that is sensitive to the operating frequency, the transmitter and receiver heights, the horizontal distance between them, and three other parameters depicting the statistical properties of the urban environment. The model developed is accurate enough to capture scattering mechanisms such as reflection and diffraction, while being sufficiently flexible mathematically to allow, based for instance on powerful analytical tools such as stochastic geometry, a seamless and physically meaningful system-level performance evaluation of modern wireless networks in the presence of airborne platforms.
\end{abstract}

Index Terms-Coverage Probability, High Altitude Platform Station (HAPS), Stochastic Geometry, Unmanned Aerial Vehicle (UAV).

\section{INTRODUCTION}

Outbreaks such as Covid-19 have spurred the digital transformation of modern society and increased the reliance on advanced mobile communication services, such as video streaming/conferencing and augmented/virtual reality (AR/VR). This trend is expected to be addressed by the fifth generation $(5 \mathrm{G})$ wireless networks, or even beyond $5 \mathrm{G}$ (B5G) that would unlock the full and sustainable potential of the Internet of Things (IoT). As of today, the bulk of 5G deployments around the world have leveraged the mid-band spectrum (i.e. 3.3 to $5 \mathrm{GHz}$ ); however, as the adoption of $5 \mathrm{G}$ evolves and more diverse devices and services mature into such networks, there will be a need for spectrum in the low (i.e. $700 \mathrm{MHz}-2.6 \mathrm{GHz}$ ) and high (i.e. 24-43.5 GHz) bands, as specified in the Third Generation Partnership Project Release 15 (3GPP-R15) and the requirements of IMT-2020 [1].

Copyright (c) 2015 IEEE. Personal use of this material is permitted. However, permission to use this material for any other purposes must be obtained from the IEEE by sending a request to pubs-permissions@ @ieee.org. Y. Hmamouche and S. Saoudi are with the Mathematical and Electrical Engineering Department, IMT-Atlantique, Lab-STICC, UMR CNRS 6285, F-29238, France (e-mail: \{yassine.hmamouche, samir.saoudi\}@imtatlantique.fr).

M. Benjillali is with the Communication Systems Department, INPT, Rabat 10100, Morocco (e-mail: benjillali@ieee.org).
In addition, the role of airborne platforms in modern wireless networks is rapidly growing and emerging as a key enabler for $5 \mathrm{G} / \mathrm{B} 5 \mathrm{G}$ communication requirements [2]. Indeed, 3GPP has considered aerial platforms as a new radio access for $5 \mathrm{G}$ as embodied in the standardization documents TR 38.811 [3], TR 22.829 [4], and TS 22.125 [5]. In this way, unmanned aerial vehicles (UAVs) operating at low altitudes of a few hundred meters can act as flexible and agile relays, base stations (BSs), or user equipments (UEs) (e.g., cargo delivery, aerial surveillance, IoT data gathering), while high-altitude platform stations (HAPSs) operating at higher altitudes of 8 to $50 \mathrm{~km}$ above the ground can offer longer flight times compared to UAVs, wider coverage areas, and transmission links between BSs and the core network for the backhauling traffic [6]. In fact, owing to their 3D mobility and scalable altitude, the statistical characteristics of the AtG channel are essentially different from ground communication channels. In this way, the evaluation of the link quality between airborne platforms and ground nodes in a range of urban propagation environments and frequency bands adopted by 3GPP-R15 and IMT-2020 is crucial for the design of 5G/B5G systems. That is, as the likelihood of LoS links is an essential component in the modeling of AtG channels [7], characterizing an accurate but tractable LoS probability (i.e., $\mathrm{P}_{\mathrm{LoS}}$ ) is a timely and ongoing critical issue in the field of system-level performance analysis of modern airborne platform-assisted wireless networks.

The modeling and calculation of the LoS probability in urban environments has been studied by both academia and standardization bodies. There are typically three families of methods: i) empirical methods that conceive the mean LoS probability using curve fitting from real-world measurement data [8]. Significant efforts based on this approach can be found in 3GPP TR 38.901 [9] and ITU-R Rec. M.2412-0 [10] and subsequently in 3GPP TR 36.777 [11] by way of extension to the context of aerial UEs; ii) deterministic methods [12] where environmental clutters are placed in specific configurations and simulation tools such as ray-tracing are performed to estimate the effects of path loss and shadowing, and thus predict the realistic behavior of electromagnetic wave propagation in these clutter setups. This method generally leads to relatively simple analytical expressions of the LoS probability, but their accuracy is dependent on the environment-specific database such as information about terrain topography, electrical parameters of buildings, and their internal structure, which renders the generalization of the model to other environments presenting different properties less precise; and iii) stochastic methods that grasp the characteristics of the propagation environment through a series of random processes whose key 
parameters are estimated from standard field measurements; this method allows to capture the key dependencies of the propagation environment along with the network performance connectivity, while also improving computational capability as compared to previous methods.

Although empirical and deterministic methods can provide a good understanding of signal propagation characteristics in the presence of airborne platforms, they remain data-driven and require extensive simulations for each new configuration of the environment. Accordingly, stochastic methods for the modeling of LoS probability emerge as a preferable choice for system-level performance analysis. Early studies, in this regard, commonly suggest that the LoS probability is a function of the statistical modeling of environmental blockages and the elevation angle between the aerial platform and the terrestrial node [13]-[18], [20], [21]. Nevertheless, the sensitivity of the LoS probability to the transmission frequency is driven by the underlying abstraction for the definition of the LoS link. Accordingly, these prior efforts can be divided into two classes:

- Models that define the LoS link by the optical ray joining the transmitter (Tx) and the receiver (Rx) [13]-[19], which implicitly assumes an almost infinite transmission frequency (since the beam width is inversely proportional to the frequency) that is not the case for the frequencies adopted by the 3GPP-R15 and IMT-2020 requirements. This LoS abstraction means that environmental blockages should not touch the direct line between $\mathrm{Tx}$ and $\mathrm{Rx}$ for the link to work properly (i.e., communication with a path loss exponent (PLE) approaching 2), but this is only partially valid. Indeed, although these standard models are generally easy-to-use and analytically flexible, they are only relatively accurate since they do not capture the scattering mechanisms such as reflection and diffraction. One important consideration for these scattering mechanisms is the intrusion degree of environmental blockages into the first Fresnel zone being defined by an ellipsoid with foci at Tx and Rx such that the path length difference between the direct path and the alternative paths is half the wavelength.

- Models that construct LoS links based on the intrusion ratio of blockages into the 1st Fresnel zone [20], [21]. Although these models can provide an accurate LoS probability that depends on the operating frequency, their resulting analytical expressions are computationally very complicated and cannot be used to develop tractable representations for important metrics of interest. In particular, such elaborate LoS probability expressions preclude a more thorough system-level performance evaluation of airborne platform-assisted communications. Actually, considering the abstraction under a powerful analytical tool such as stochastic geometry [22], these models fail to derive the following integral

$$
\Lambda(u)=\int_{0}^{u} x\left(1-\mathrm{P}_{\mathrm{LoS}}(x)\right) \mathrm{d} x,
$$

which is necessary to obtain, based on the concept of independent thinning of the Poisson point process (PPP)
[22], the spatial intensity of nodes in LoS and non lineof-sight (NLoS) conditions with the typical UE, and then to calculate the probability density function (PDF) of the horizontal distance between the typical UE and the nearest node in LoS and NLoS transmission. These PDFs are in turn essential for the evaluation of key performance metrics, such as coverage probability and ergodic rate.

Overall, tractable LoS probability models are actually less realistic since they abstract the required LoS area based on the direct ray joining $\mathrm{Tx}$ and $\mathrm{Rx}^{1}$, while accurate models based on the intrusion ratio into the 1st Fresnel ellipsoid are computationally intensive, intractable, and cannot be exploited for a thorough system-level performance evaluation of airborne platform-assisted communications. In this paper, we aim to bridge this gap and overcome the limitations of stochastic approaches by developing a LoS probability model that offers a good tradeoff between modelling accuracy and analytical tractability. The main contributions of this work can be summarised as follows.

- Using tools from the two-ray propagation and the knife edge diffraction (KED) models, we first demonstrate that the key issue affecting the transition from free-space path loss (FSPL), i.e., PLE $\simeq 2$, to a much stronger one, i.e., $\mathrm{PLE}>2$, or equivalently the transition from LoS to NLoS conditions, is indeed the intrusion degree of environmental objects into the 1st Fresnel zone, rather than into the optical ray joining Tx and Rx.

- Considering the statistical model of blockages adopted by the ITU-R Rec. P.1410-5 [24], we propose a stretched exponential function for the probability of LoS by building the required LoS clearance zone on a fraction of the 1 st Fresnel zone. Results show that the proposed model can outperform the popular and widely adopted model [13] in terms of accuracy and analytical tractability.

- Taking benefit from the improved accuracy and tractability of the proposed LoS model; we illustrate its applicability by analyzing the coverage probability at a UE of interest served by a particular aerial platform. The developed LoS probability enables to derive a closed-form expression of the PDF of the horizontal distance between the typical ground UE and the best aerial platform in terms of the lowest path loss association scheme. Using tools from stochastic geometry [22], we are able to evaluate the coverage probability for both fixed/deterministic and random PPP-distributed UAV/HAPS locations around the typical ground UE.

Notations: $\mathbb{P}(\cdot)$ and $\mathbb{E}(\cdot)$ stand for probability and expectation measures, $F_{X}(\cdot)$ is the cumulative distribution function (CDF) of a random variable $X, \Gamma(s, x)=\int_{x}^{\infty} t^{s-1} \mathrm{e}^{-t} \mathrm{~d} t$ is the upper incomplete gamma function, while $\Gamma(s, 0)=\Gamma(s)$ is

\footnotetext{
${ }^{1}$ In some mission-critical tasks, this lack of accuracy can actually be problematic as it may distort the evaluation of system-level performance. In fact, it tends to overestimate the likelihood of LoS for the desired serving Tx and thus leads to an optimistic version of the signal-to-noise ratio (SNR). Alternatively, it can overestimate the detrimental effect of interference (since blockages can be seen as a natural mechanism for interference avoidance [23]) and thus lead to a pessimistic version of the signal-to-interference-plus-noise ratio (SINR).
} 


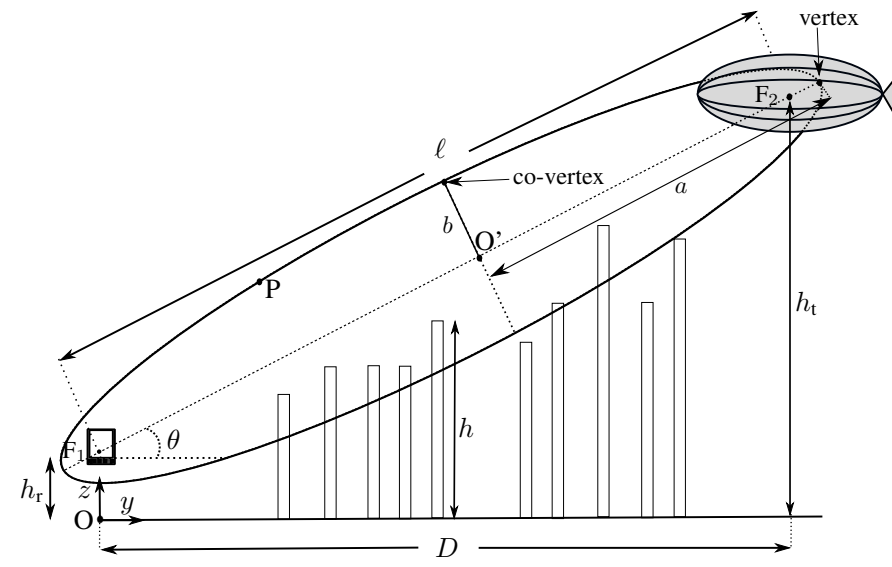

Fig. 1. Geometry of the 1st Fresnel zone clearance.

the ordinary gamma function, and $\min (x, y)$ and $\max (x, y)$ are the minimum and the maximum of two reals $x$ and $y$, respectively. $|A B|$ is the Euclidean distance between points A and $\mathrm{B}$.

\section{INTERPRETATION OF THE REQUIRED LINE-OF-SIGHT}

In this section, leveraging the resilience of the two-ray propagation and KED models, we establish analytically and numerically that the crucial factor affecting the transition from LoS to NLoS in AtG connections is actually the intrusion ratio of obstacles into the 1st Fresnel ellipsoid.

\section{A. Blockages Setup}

As depicted in Fig. 1, we consider a single-link communication scenario between an aerial Tx (i.e., aerial platform) located at an altitude $h_{\mathrm{t}}$ and a ground Rx (i.e., UE) located at an altitude $h_{\mathrm{r}}$. The elevation angle is denoted by $\theta$, while UE and the aerial platform are separated by a distance $\ell=\sqrt{D^{2}+\Delta h_{\mathrm{t}, \mathrm{r}}^{2}}$, where $D$ is the horizontal distance between Tx and $\mathrm{Rx}$ and $\Delta h_{\mathrm{t}, \mathrm{r}}=h_{\mathrm{t}}-h_{\mathrm{r}}$ is the height difference between Tx and Rx.

We adopt the statistical setup of the ITU-R Rec. P.14105 [24] in which the buildings are assumed to have null width and are evenly spaced along the propagation direction between Tx and Rx. In such a context, the number of buildings between Tx and Rx can be tuned by two key parameters: i) $\alpha$ [dimensionless] the ratio of land area covered by buildings to total land area, and ii) $\beta$ [number of buildings $/ \mathrm{km}^{2}$ ] the average number of buildings per unit area ${ }^{2}$. The number of buildings between Tx and Rx separated by a horizontal distance $D$ in [m] is expressed as [24]

$$
N(D)=\lfloor D \sqrt{\alpha \beta} / 1000\rfloor,
$$

while the horizontal distance between $\mathrm{O}$ and the $k$ th building is expressed as [24]

$$
y_{k}=(k-0.5) D / N(D), \text { for } k=1, \ldots, N(D) .
$$

\footnotetext{
${ }^{2} \beta$ is introduced to distinguish between propagation environments where the buildings structure generates the same $\alpha$ but with a different spatial arrangement.
}

Furthermore, blockages are assumed to be generated with random heights based on a given PDF $f_{h}(\cdot)$. Note that the Rayleigh distribution is adopted by the ITU-R Rec. P.1410-5 [24], where the probability that the building height is equal to $u$ is expressed in terms of the most frequent building height $\gamma$ as

$$
f_{h}(u)=\frac{u}{\gamma^{2}} \exp \left(-\frac{u^{2}}{2 \gamma^{2}}\right) .
$$

Typical values of $\gamma, \alpha$, and $\beta$ enabling to capture different urban propagation environments from suburban to highrise urban areas can be found in [18]. In particular, the triples $(\gamma, \alpha, \beta)$ are calculated as $(0.1,750,8)$ for suburban, $(0.3,500,15)$ for urban, $(0.5,300,20)$ for dense urban, and $(0.5,300,50)$ for high-rise urban.

If there are no environmental obstructions (building, tree, hill, etc.) between Tx and Rx, radio waves travel in a straight line between them. Otherwise, they can reflect off these surfaces and arrive either out of phase or in phase with the signals going directly to the Rx. One important consideration to evaluate propagation losses due to diffraction and reflection brought by objects between the Tx and Rx is the concept of the 1st Fresnel zone [25]. It is defined as an ellipsoid whose foci are located at Tx and Rx, where the path length between the direct path and the alternative paths is half-wavelength. As illustrated in Fig. 1, the 1st Fresnel ellipsoid centered at O', can be constructed as the locus of all points $\mathrm{P}$ for which the sum of the distances from the antennas Rx (i.e. point F1) and Tx (i.e. point F2) is half a wavelength greater than the direct distance between Tx and Rx. Formally,

$$
\left|F_{1} P\right|+\left|P F_{2}\right|=\ell+\frac{\lambda}{2},
$$

where $\lambda=c / f$ is the wavelength such that $c$ is the speed of light and $f$ is the operating frequency.

As a special case, the semi-major axis $a$ of the ellipse can be obtained from (5) by considering $\mathrm{P}$ at one vertex of the ellipse, which gives

$$
2 a=\ell+\frac{\lambda}{2} .
$$

Also, the semi-minor axis $b$ of the ellipse can be obtained from (5) by considering $\mathrm{P}$ at one co-vertex of the ellipse, which gives

$$
2 \sqrt{b^{2}+\frac{\ell^{2}}{4}}=\ell+\frac{\lambda}{2} .
$$

As a numerical application, consider a transmission frequency of $2.4 \mathrm{GHz}$ and a distance $\ell=1 \mathrm{~km}$. The semi-minor axis $b$ can be estimated using (7) resulting in $5.6 \mathrm{~m}$, which is much smaller than the dimensions of a typical building. In such scenarios, evaluating the LoS probability through the likelihood of impinging obstacles within the 1st 2D Fresnel ellipse does make sense. For analytical tractability, the generalization of the analysis through a 3D ellipsoid as in [20], [21] will be studied in a future work.

Accordingly, the equation of the 1st Fresnel ellipsoid in (Oyz) plane can be obtained based on some transformations 
(i.e., rotation counterclockwise through $\theta$ and translation), as [20], [21]

$$
\begin{aligned}
& \frac{\left(y \cos (\theta)+\left(z-h_{\mathrm{r}}\right) \sin (\theta)-\frac{\ell}{2}\right)^{2}}{a^{2}} \\
& \quad+\frac{\left(\left(z-h_{\mathrm{r}}\right) \cos (\theta)-y \sin (\theta)\right)^{2}}{b^{2}}=1,
\end{aligned}
$$

where $a$ and $b$ are, respectively, the semi-major and semiminor axis of the ellipse expressed from (6) and (7) as $a=$ $\frac{\ell}{2}+\frac{\lambda}{4}, b=\frac{1}{2} \sqrt{\lambda\left(\ell+\frac{\lambda}{4}\right)}, \theta$ is the angle of elevation in radians obtained as $\theta=\arctan \left(\frac{\Delta h_{\mathrm{t,r}}}{D}\right)$.

As depicted in Fig. 1, it can be observed that the top of an obstructing object can intrude into the 1st Fresnel zone without cutting the optical ray joining the Tx and Rx antennas. In this case, a component of the signal is reflected from the top of the object to create an alternate path to the receiver. Thus, the direct path and the reflected one have different path lengths, resulting in different phases of the signals traveling along the two paths at the receiver, so they combine constructively or destructively to increase or decrease the signal strength at the receiver. Alternatively, if the obstruction were to be higher and intrude deeper into the Fresnel zone to intersect with the optical ray between $\mathrm{Tx}$ and $\mathrm{Rx}$, we would no longer have a direct transmission path, nor a conventional reflective surface to produce an alternative signal path. Instead, we would have another physical phenomenon; namely, diffraction. Overall, it is important to keep the 1st Fresnel zone clear enough in order to operate the communication under an FSPL (i.e., establishing a LoS connection). In the following, we will discuss the impact of the intrusion ratio of blockages into the 1st Fresnel zone on the establishment of a LoS connection between Tx and Rx.

\section{B. Two-Ray Propagation Model}

We consider a two-ray propagation model [25] which, based on geometric optics, will capture a direct ray and a groundreflected ray between $\mathrm{Tx}$ and $\mathrm{Rx}$, as illustrated in Fig. 2(a). Assuming a specular reflection by the ground, the received signal strength (RSS) at the UE of interest can be expressed as a function of the transmit power $P_{\mathrm{t}}$ as [25]

$$
\mathrm{RSS}=P_{\mathrm{t}}\left(\frac{\lambda}{4 \pi}\right)^{2}\left|\frac{1}{\ell}-\frac{\mathrm{e}^{-j \Delta \phi}}{r_{1}+r_{2}}\right|^{2}
$$

where $j^{2}=-1, r_{1}+r_{2}=\sqrt{D^{2}+\left(h_{\mathrm{r}}+h_{\mathrm{t}}\right)^{2}}$ is the length of the reflected path on the ground, and $\Delta \phi=\frac{2 \pi\left(r_{1}+r_{2}-\ell\right)}{\lambda}$ is the phase difference between the direct and the reflected paths.

Note that for sufficiently small $\ell$, the ground reflection is not possible according to Snell's law of reflection and thus the Tx-Rx connection is established via the direct path only. In this way, RSS in (9) scales with $\ell^{-2}$. For sufficiently large $\ell$, relatively to the height of $\mathrm{Tx} / \mathrm{Rx}$ antennas, we have $\ell \simeq r_{1}+r_{2}$ and then $\mathrm{e}^{-j \Delta \phi} \simeq 1-j \Delta \phi \simeq 1-j \frac{4 \pi h_{\mathrm{t}} h_{\mathrm{r}}}{\lambda D}$, RSS in (9) starts then to scale with $\ell^{-4}$. Similarly, as the distance between Tx and Rx increases and/or the transmission frequency reduces (i.e., $\lambda$ increases), $b$ becomes larger, which increases the probability that the 1st Fresnel zone and/or inner

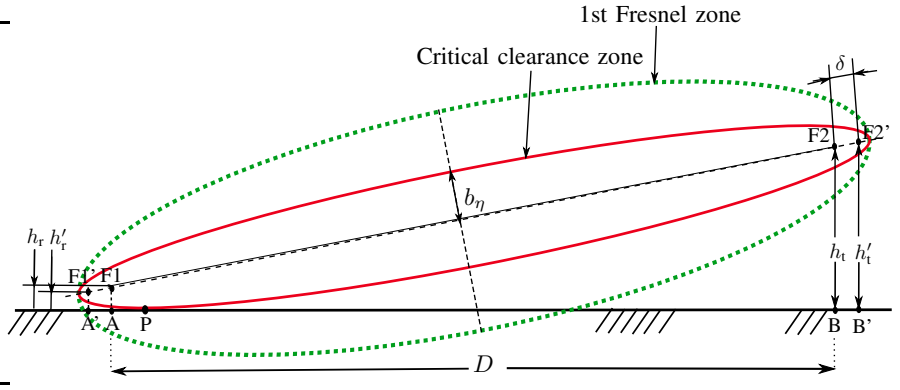

(a)

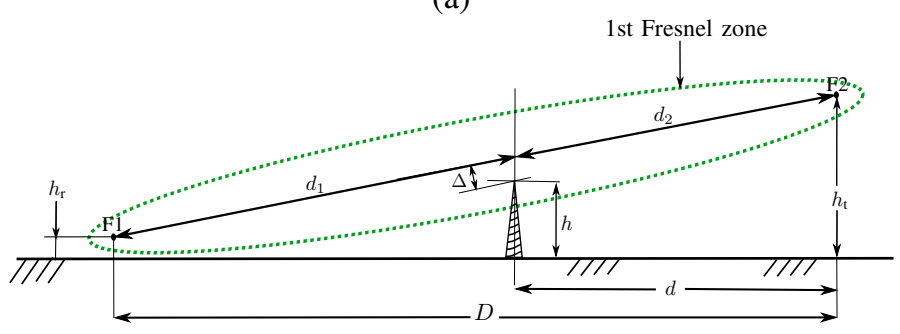

(b)

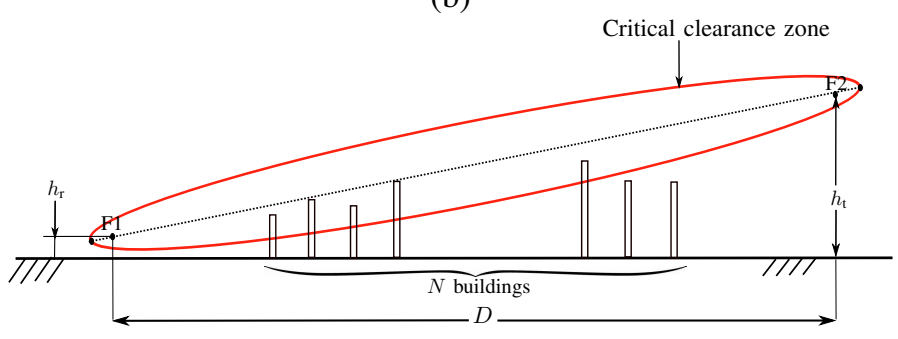

(c)

Fig. 2. (a) Abstraction of a two-ray propagation model, where the dotted ellipse indicates the 1st Fresnel zone, with two foci F1 (located at the UE) and F2 (located at the aerial platform). The inner solid line ellipse represents the required crucial area of LoS clearance, where $b_{\eta}=\eta b$ is the length of the semi-minor axis such that $0<\eta \leq 1$, and foci F1' and F2' displaced from F1 and F2 such that $\delta=\sqrt{a^{2}-\eta^{2} b^{2}}-\sqrt{a^{2}-b^{2}}$, (b) Penetration of a knife-edge obstacle into the 1st Fresnel zone, (c) Regular separation distance between buildings in a built-up area.

ellipses (i.e., ellipses with a semi-minor axis length $b_{\eta}=\eta b$ with $0<\eta \leq 1$ ) will hit the ground plane, and then induce fluctuations in the propagation losses.

We aim now to evaluate the interplay between the crossing point of the RSS scaling range from $\ell^{-2}$ to $\ell^{-4}$ and the penetration ratio of the ground into the 1st Fresnel zone. Let's consider the scenario where the 1st Fresnel zone intrudes into the ground (which might be possible by acting on $D, \lambda$, and $\left.\Delta h_{\mathrm{t}, \mathrm{r}}\right)$, so that the inner ellipse with semi-minor axis length $b_{\eta}$ just starts to reach the ground at a point P (cf. Fig. 2(a)). The rationale is to evaluate up to what value of $\eta$, obstructions commencing to encroach on this inner ellipse (i.e. the critical clearance zone in Fig. 2) will trigger the transition of the RSS scaling range from $\ell^{-2}$ to $\ell^{-4}$.

From the geometrical construction of inner ellipses, point $\mathrm{P}$ verifies that

$$
\begin{aligned}
\left|F 1^{\prime} P\right|+\left|P F 2^{\prime}\right| & =2 a \\
\sqrt{{h_{\mathrm{r}}^{\prime}}^{2}+\left|A^{\prime} P\right|^{2}}+\sqrt{{h_{\mathrm{t}}^{\prime}}^{2}+\left|P B^{\prime}\right|^{2}} & =\ell+\frac{\lambda}{2} .
\end{aligned}
$$


Then, since

$$
\left\{\begin{array}{c}
h_{\mathrm{t}}^{\prime}\left|A^{\prime} P\right|=h_{\mathrm{r}}^{\prime}\left|P B^{\prime}\right| \\
\left|A^{\prime} P\right|+\left|P B^{\prime}\right|=\left|A^{\prime} B^{\prime}\right|
\end{array},\right.
$$

and

$$
\left\{\begin{array}{l}
h_{\mathrm{t}}^{\prime}=h_{\mathrm{t}}+\delta \sin (\theta)=\left(1+\frac{\delta}{\ell}\right) h_{\mathrm{t}}-\frac{\delta}{\ell} h_{\mathrm{r}} \\
h_{\mathrm{r}}^{\prime}=h_{\mathrm{r}}-\delta \sin (\theta)=\left(1+\frac{\delta}{\ell}\right) h_{\mathrm{r}}-\frac{\delta}{\ell} h_{\mathrm{t}}
\end{array},\right.
$$

(10) can be simplified as

$$
\left|A^{\prime} B^{\prime}\right|^{2}+\left(h_{\mathrm{t}}+h_{\mathrm{r}}\right)^{2}=\left(\ell+\frac{\lambda}{2}\right)^{2}
$$

where

$$
\begin{aligned}
\left|A^{\prime} B^{\prime}\right|^{2} & =(\ell+2 \delta)^{2}-\left(h_{\mathrm{t}}^{\prime}-h_{\mathrm{r}}^{\prime}\right)^{2} \\
& \stackrel{(a)}{=}(\ell+2 \delta)^{2}-\left(h_{\mathrm{t}}-h_{\mathrm{r}}\right)^{2}\left(1+\frac{2 \delta}{\ell}\right)^{2},
\end{aligned}
$$

where (a) follows from (12). Combining (13) and (14) gives

$(\ell+2 \delta)^{2}+\left(h_{\mathrm{t}}+h_{\mathrm{r}}\right)^{2}=\left(\ell+\frac{\lambda}{2}\right)^{2}+\left(h_{\mathrm{t}}-h_{\mathrm{r}}\right)^{2}\left(1+\frac{2 \delta}{\ell}\right)^{2}$.

Considering that the direct link distance is generally very much larger than the wavelength, i.e., $\ell \gg \lambda, \delta$ can be derived as

$$
\begin{aligned}
\delta & =\sqrt{a^{2}-\eta^{2} b^{2}}-\sqrt{a^{2}-b^{2}} \\
& \stackrel{(a)}{=} \frac{\ell}{2}\left(\sqrt{\left(1+\frac{\lambda}{2 \ell}\right)^{2}-\frac{\lambda \eta^{2}}{\ell}\left(1+\frac{\lambda}{4 \ell}\right)}-1\right) \\
& \stackrel{(b)}{\simeq} \frac{\ell}{2}\left(\sqrt{1+\frac{\lambda\left(1-\eta^{2}\right)}{\ell}}-1\right) \\
& \stackrel{(c)}{\simeq} \frac{\lambda\left(1-\eta^{2}\right)}{4},
\end{aligned}
$$

where (a) is obtained by replacing the semi-major axis $a$ and the semi-minor axis $b$ by their respective expressions in (6) and (7), (b) follows by ignoring the second order of $(\lambda / \ell)$, and (c) holds since $(1+x)^{n} \simeq 1+n x$ for very small $x \forall n \in \mathbb{N}$.

(15) can then be simplified as

$$
\ell^{2}-\frac{4 h_{\mathrm{t}} h_{\mathrm{r}}}{\lambda \eta^{2}} \ell+\frac{\left(1-\eta^{2}\right)\left(h_{\mathrm{t}}-h_{\mathrm{r}}\right)^{2}}{\eta^{2}}=0 .
$$

In this way, the horizontal distance $D$ between Tx and Rx can be calculated as

$$
D=\sqrt{\ell^{2}-\left(h_{\mathrm{t}}-h_{\mathrm{r}}\right)^{2}}
$$

where $\ell$ is the positive root from (17), resulting in

$$
\ell=\frac{2 h_{\mathrm{t}} h_{\mathrm{r}}}{\lambda \eta^{2}}+\sqrt{\left(\frac{2 h_{\mathrm{t}} h_{\mathrm{r}}}{\lambda \eta^{2}}\right)^{2}-\left(\frac{1-\eta^{2}}{\eta^{2}}\right)\left(h_{\mathrm{t}}-h_{\mathrm{r}}\right)^{2}} .
$$

Fig. 3 calculates the path loss between Tx and Rx under the two-ray propagation model for different key $5 \mathrm{G}$ NR spectrum bands (i.e., $700 \mathrm{MHz}, 3.5 \mathrm{GHz}$, and $25 \mathrm{GHz}$ ). Table I calculates analytically the horizontal distance $D$ from (18) for $\eta=0.6$ and 1. Mapping these analytical results of $D$ with the numerical ones in Fig. 3 shows that although one may initially think that the 1st Fresnel zone boundary might be the starting

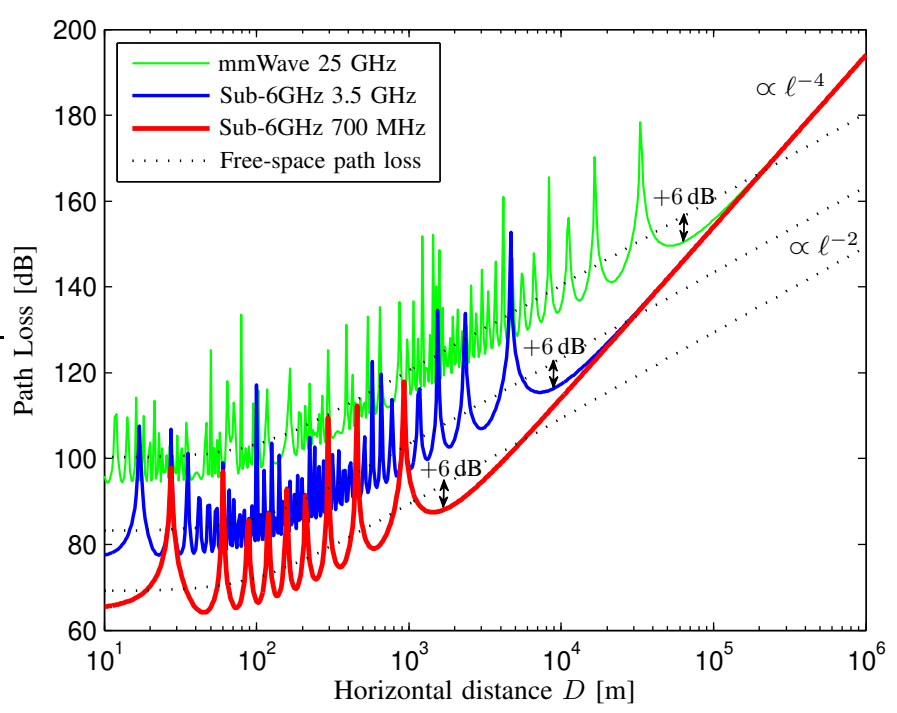

Fig. 3. Calculation of the path loss under the two-ray propagation model exploiting different key 5G NR spectrum bands for comparison with the FSPL model. The height of the UE and aerial platform are set to $h_{\mathrm{r}}=2 \mathrm{~m}$ and $h_{\mathrm{t}}=100 \mathrm{~m}$, respectively.

TABLE I

ANALYTICAL RESULTS FOR $D$

\begin{tabular}{|l|l|l|l|}
\hline$\eta$ & $700 \mathrm{MHz}$ & $3.5 \mathrm{GHz}$ & $25 \mathrm{GHz}$ \\
\hline \hline 1 & $1.86 \mathrm{~km}$ & $9.33 \mathrm{~km}$ & $66.66 \mathrm{~km}$ \\
\hline 0.6 & $5.18 \mathrm{~km}$ & $25.92 \mathrm{~km}$ & $185.19 \mathrm{~km}$ \\
\hline
\end{tabular}

point for signal attenuation, it actually appears to be a place where the signal is being amplified. Indeed, for $\eta=100 \%$ (i.e., $0 \%$ ground intrusion), we obtain approximately $+6 \mathrm{~dB}$ gain w.r.t. FSPL. Besides, when the ground starts to intrude into the 1st Fresnel zone (i.e., $\eta$ is decreasing), the $+6 \mathrm{~dB}$ gain starts to decrease, until it becomes $0 \mathrm{~dB}$ when $\eta$ is approximately around $60 \%$ (cf. Fig. 3). In other words, when the ground starts to penetrate nearly into the $60 \%$ region of the 1st Fresnel zone, this is a trigger factor leading to the change in the PLE from 2 to 4 . So, by enlarging the 1st Fresnel zone either by increasing the horizontal distance between Tx and Rx or by lowering the transmission frequency, the NLoS transmission starts occurring from the horizontal distance $D$ enabling $\eta=0.6$ even though there is a clear visibility between Tx and Rx.

\section{Knife Edge Diffraction Model}

Assuming ideal conditions of LoS propagations where there are no environmental objects between the Tx and Rx, the tworay model of geometrical optics can provide accurate path loss results in Fig. 3. However, this technique is not suitable to evaluate the scenario where diffraction by environmental objects is significant. In other words, those events where the blockage may come very close to the optical ray joining $\mathrm{Tx}$ and Rx, or even cross it (cf. Fig. 2(b)). In such a context, the KED model is widely adopted for approximate prediction of diffraction losses from a single edge in outdoor propagation environments, as well outlined in the ITU-R Rec. P.526-15 [26]. According to this theory, the total path loss over a single 


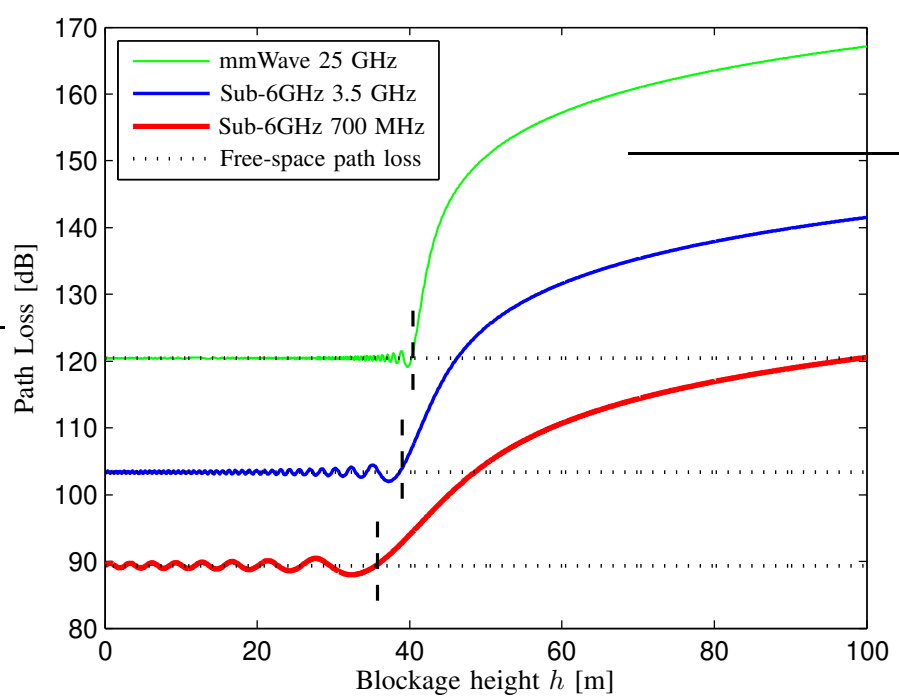

Fig. 4. Variations of the total path loss as a function of the transmission frequency and height of the penetrating blockage located at a distance $d=$ $600 \mathrm{~m}$ from the Tx. The Tx-Rx parameters are $h_{\mathrm{t}}=100 \mathrm{~m}, h_{\mathrm{r}}=2 \mathrm{~m}$, and $D=1000 \mathrm{~m}$.

impenetrable knife-edge obstacle can be expressed as follows

$$
P L=F S P L+P L_{\text {diff }}
$$

where $\mathrm{PL}_{\text {diff }}$ is the diffraction loss due to the intrusion of the knife-edge obstacle into the 1st Fresnel zone. It is expressed as

$\mathrm{PL}_{\mathrm{diff}}=-20 \log _{10}\left(\frac{\sqrt{(1-C(v)-S(v))^{2}+(C(v)-S(v))^{2}}}{2}\right)$,

where $C(v)$ and $S(v)$ are the real and imaginary parts of the complex Fresnel integral $F_{\mathrm{c}}(v)=\int_{0}^{v} \exp \left(j \frac{\pi s^{2}}{2}\right) \mathrm{d} s$, respectively, and $v$ is a dimensionless parameter expressed as a function of the terms defined in Fig. 2(b) as

$$
v=\Delta \sqrt{\frac{2}{\lambda}\left(\frac{1}{d_{1}}+\frac{1}{d_{2}}\right)} .
$$

Note that $\Delta$ is measured w.r.t. straight line joining the two ends of the path. It is positive if the top of the obstruction cuts through the direct path, and negative if it is below it. Using some geometric manipulations in Fig. 2(b), we can obtain

$$
\begin{aligned}
\Delta & =\frac{\left(h-h_{\mathrm{t}}\right) D+\left(h_{\mathrm{t}}-h_{\mathrm{r}}\right) d}{\sqrt{D^{2}+\left(h_{\mathrm{t}}-h_{\mathrm{r}}\right)^{2}}}, \\
\frac{d_{1} d_{2}}{d_{1}+d_{2}} & =\frac{d}{D}\left(1-\frac{d}{D}\right) \sqrt{D^{2}+\left(h_{\mathrm{t}}-h_{\mathrm{r}}\right)^{2}} .
\end{aligned}
$$

In Fig. 4, we plot the fluctuations of the total path loss in (20) as a function of the degree of blockage intrusion into the 1st Fresnel zone (Fig. 2(b)). Typically, the minimum height of the knife-edge obstacle causing diffraction losses can be calculated numerically from Fig. 4. In particular, we have $h=35.2 \mathrm{~m}$ at $700 \mathrm{MHz}, h=38.5 \mathrm{~m}$ at $3.5 \mathrm{GHz}$, and

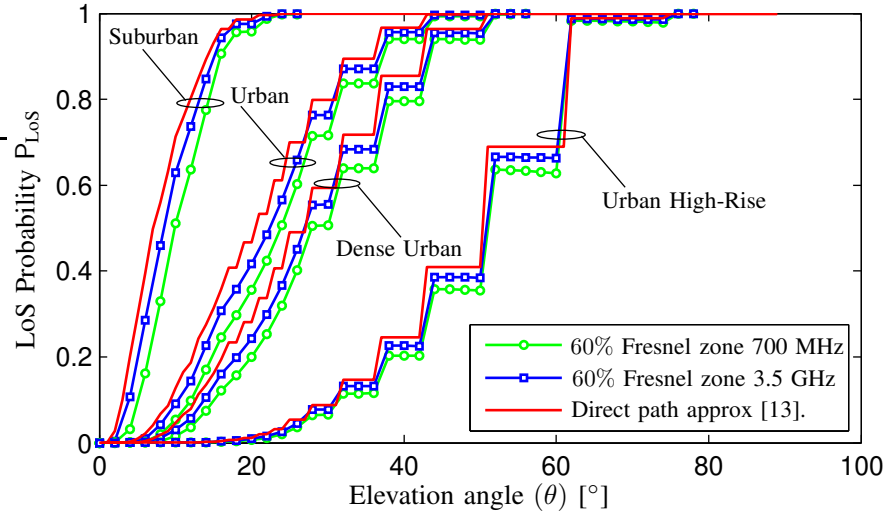

(a)

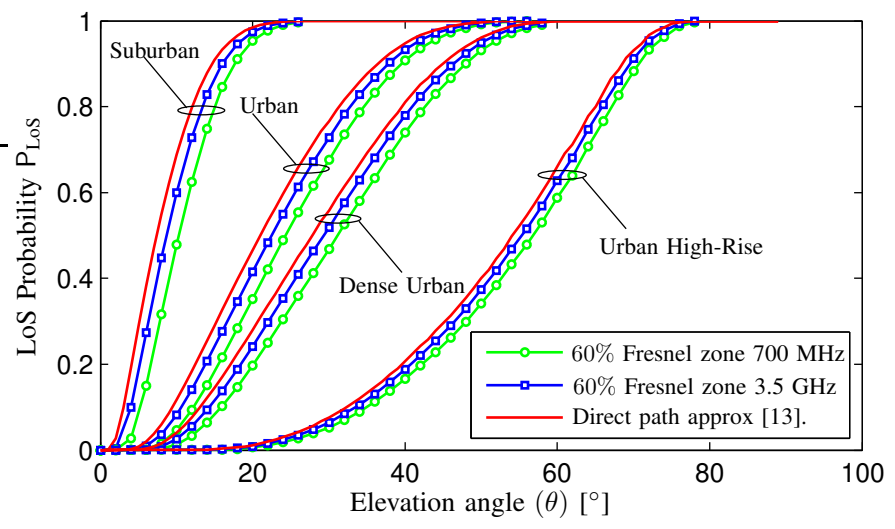

(b)

Fig. 5. Calculated LoS probability under the direct link and 60\% Fresnel zone abstractions for different propagation environments and transmission frequencies. Tx-Rx height parameters: $h_{\mathrm{r}}=2 \mathrm{~m}$ and (a) $h_{\mathrm{t}}=300 \mathrm{~m}$, (b) $h_{\mathrm{t}}=10 \mathrm{~km}$.

$h=40.2 \mathrm{~m}$ at $25 \mathrm{GHz}$. Given that the radius of the 1 st Fresnel zone at the point where the knife-edge obstacle intrudes is $r_{\mathrm{d}}=\sqrt{\lambda \frac{d_{1} d_{2}}{d_{1}+d_{2}}}$ (which simplifies as $r_{\mathrm{d}} \simeq 241.15 \sqrt{\lambda}$ for the numerical values of Fig. 4 in (24)). Previous numerical results of $h$ yield $\Delta / r_{\mathrm{d}} \simeq 0.6$, which is equivalent to almost $60 \%$ of obstacle intrusion into the 1st Fresnel zone.

From previous discussions, it is clear that the definition of a LoS link needs to be judiciously based on the intrusion ratio of obstacles into the 1st Fresnel zone between Tx and Rx rather than on the simplistic fact of cutting the direct ray joining them [13]. In this way, we develop in the next section an accurate but tractable LoS probability model. Accurate since it is based on the intrusion ratio of environmental blockages into the 1st Fresnel zone rather than the direct line between Tx and Rx; and tractable since it enables the derivation of closedform expressions with PDFs of interest, which facilitates a thorough system-level performance evaluation of airborne platform-assisted communications.

\section{Modeling The Line-OF-Sight Probability}

As illustrated in Fig. 2(c), for a given number $N(D)$ of buildings between Tx and Rx, the probability of LoS is defined as the probability that the height $h_{k}(k=1, \ldots, N(D))$ of all blockages between $\mathrm{Tx}$ and $\mathrm{Rx}$ is smaller than $z_{k}$, the least solution of (8) for $y \rightarrow y_{k}=(k-0.5) D / N(D)$ (i.e., 


$$
\begin{aligned}
z_{k}=h_{\mathrm{r}}+\frac{1}{2\left(a^{2} \cos ^{2}(\theta)+b_{\eta}^{2} \sin ^{2}(\theta)\right)} & \left(y_{k} \sin (2 \theta)\left(a^{2}-b_{\eta}^{2}\right)+\ell b_{\eta}^{2} \sin (\theta)\right. \\
& \left.-2 a b_{\eta} \sqrt{\frac{\cos (2 \theta)}{2}\left(a^{2}-b_{\eta}^{2}-\frac{\ell^{2}}{4}\right)+\frac{1}{2}\left(a^{2}+b_{\eta}^{2}-\frac{\ell^{2}}{4}\right)+y_{k}\left(\ell \cos (\theta)-y_{k}\right)}\right) .
\end{aligned}
$$

\section{TABLE II}

KEY STOCHASTIC LOS PROBABILITY MODELS FOR ELEVATED TX AND/OR RX WITH ASSOCIATED SYSTEM-LEVEL ANALYTICAL TRACTABILITY AND

\begin{tabular}{|c|c|c|c|c|c|c|c|}
\hline Reference & LoS Probability Model & Parameters & $\begin{array}{l}\text { System-level } \\
\text { analytical tractability }\end{array}$ & \multicolumn{3}{|c|}{ RMS } & $\begin{array}{l}\text { Average } \\
\text { RMS }\end{array}$ \\
\hline [13] & $\frac{1}{1+m \exp \left(-n\left(\frac{180}{\pi} \arctan \left(\frac{\Delta h_{\mathrm{t}, \mathrm{r}}}{D}\right)-m\right)\right)}$ & $m, n$ & Medium & 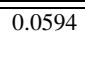 & 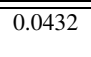 & 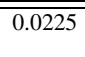 & 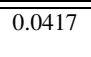 \\
\hline [18] & $m+\frac{m-n}{1+\left(\frac{\frac{180}{\pi} \arctan \left(\frac{\Delta h_{\mathrm{t}, \mathrm{r}}}{D}\right)-p}{q}\right)^{s}}$ & $m, n, p, q, s$ & Medium & 0.0622 & 0.0303 & 0.02 & 0.0375 \\
\hline [17], [19] & $\exp \left(-m n \frac{D}{\Delta h_{\mathrm{t}, \mathrm{r}}}\right)$ & $\begin{array}{l}m, n=\min \left(\mathbb{E}(h), \Delta h_{\mathrm{t}, \mathrm{r}}\right)[17] \\
\text { or } n=4 \gamma \sqrt{\frac{2 \alpha \beta}{\pi}} Q\left(\frac{h_{\mathrm{r}}}{\gamma}\right)[19]\end{array}$ & High & 0.0503 & 0.0397 & 0.0206 & 0.0369 \\
\hline Proposed & $1-\exp \left(-m\left(\frac{\Delta h_{t, \mathrm{r}}}{D}\right)^{n}\right)$ & $m, n$ & High & 0.0502 & 0.0327 & 0.0197 & 0.0342 \\
\hline
\end{tabular}
ACCURACY IN THE AVERAGE RMS SENSE.

the distance between $\mathrm{O}$ and the $k$ th building) and $b_{\eta}=\eta b$ as the length of the semi-minor axis. In other words, each building between Tx and Rx should not intrude into the critical clearance zone (e.g., $60 \%$ of the 1 st Fresnel zone for $\eta=0.6$ ). Formally,

$$
\begin{aligned}
\mathrm{P}_{\mathrm{LoS}} & =\prod_{k=1}^{N(D)} \mathbb{P}\left(h_{k}<z_{k}\right) \stackrel{(a)}{=} \prod_{k=1}^{N(D)} F_{h}\left(z_{k}\right) \\
& \stackrel{(b)}{=} \prod_{k=1}^{N(D)}\left(1-\exp \left(\frac{-z_{k}^{2}}{2 \gamma^{2}}\right)\right)
\end{aligned}
$$

where (a) holds since $\left(h_{k}\right)_{k=1, \ldots, N(D)}$ are independent and identically distributed (i.i.d.) and (b) comes from the PDF of buildings height in (4).

Solving the quadratic equation in (8) for $y \rightarrow y_{k}$ and $b \rightarrow$ $b_{\eta}$, we obtain the expression of $z_{k}$ in (26). Note that if we set $\eta \rightarrow 0$, thus considering that the abstraction of LoS is actually based on the direct line connecting $\mathrm{Tx}$ and $\mathrm{Rx}$, (26) reduces to

$$
z_{k}=h_{\mathrm{r}}+y_{k} \tan (\theta)
$$

which is independent of the transmission frequency as in [13], [24]. In this way, Fig. 5 illustrates the curve of LoS probability as a function of the elevation angle in different urban environments, highlighting the differences between the paradigms of constructing the LoS clearance zone based on the intrusion ratio into the 1st Fresnel zone (Eq. (25) based on (26)) or on the optical ray joining Tx and Rx (Eq. (25) based on (27)).

The LoS probability in (25) calculated from (26) as a series product is in a complicated form and cannot be effectively implemented in the analytical approach presented in next section. We then propose its approximation by a compact mathematical expression that is both analytically tractable and allows the best fit to empirical data in terms of the root-meansquare (RMS) deviation defined as

$$
\mathrm{RMS}=\sqrt{\sum_{i=1}^{s} \frac{\left(\mathrm{P}_{\mathrm{LoS}}^{\mathrm{c}}\left(\theta_{i}\right)-\mathrm{P}_{\mathrm{LoS}}\left(\theta_{i}\right)\right)^{2}}{s}},
$$

where $s$ is the size of data samples and $\mathrm{P}_{\mathrm{LoS}}^{\mathrm{c}}\left(\theta_{i}\right)$ and $\mathrm{P}_{\mathrm{LoS}}\left(\theta_{i}\right)$ are, respectively, the calculated and model-based data LoS probability for $1 \leq i \leq s$.

We therefore propose to build on the merits of the stretched exponential function (also known as the complementary CDF of Weibull distribution) given by $\mathrm{e}^{-m u^{n}}$, where $m$ and $n$ are tunable parameters. This model has demonstrated great success in modeling several measures of interest in wireless communications. For instance, the stretched exponential is shown to capture accurately experimental propagation measurements for dense and ultra-dense networks [27]. It also emerges in the Laplace transform for interference power when the location of the transmitters is modeled as a 2D PPP with no exclusion region around the typical receiver [28, Eq. (17)]. In this way, we propose a LoS probability model of the form

$$
\mathrm{P}_{\mathrm{LoS}}(D)=1-\exp \left(-m_{\mathrm{f}}\left(\frac{\Delta h_{\mathrm{t}, \mathrm{r}}}{D}\right)^{n_{\mathrm{f}}}\right),
$$

where $m_{\mathrm{f}}$ and $n_{\mathrm{f}}$ are positive fitting parameters.

Fig. 6 verifies this model with the same simulation results developed in [19] for different UE height and an aerial Tx at $h_{\mathrm{t}}=300 \mathrm{~m}$. Results show that owing to its mathematical construction, the proposed model in (29) can provide the best fit among all popular candidate models presented in Table II. Typically, these models are ordered by increasing improvement of the average RMS error over the three considered UE heights. In particular, and for the sake of clarity, only the two models with the highest and lowest performance in terms of average RMS are shown in Fig. 6. In fact, the simple modified Sigmoid function proposed in [13, Eq. (5)] is actually the most popular and widely used model in the literature with more 


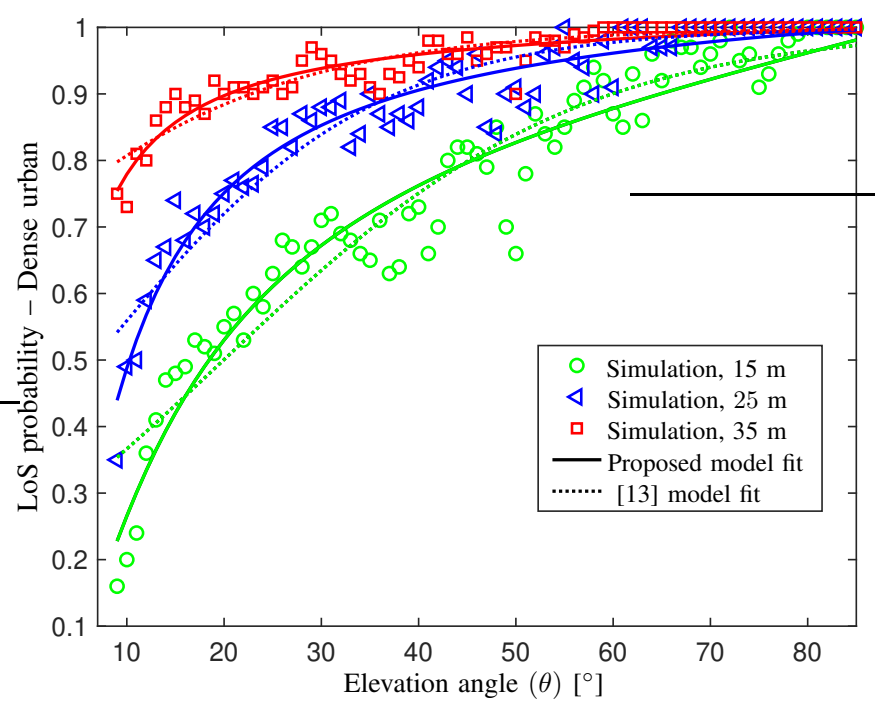

Fig. 6. LOS probability as a function of Rx height and elevation angle for $h_{\mathrm{t}}=300 \mathrm{~m}$ and $f=2.4 \mathrm{GHz}$ in dense urban environments.

than 1200 citations and, thus, will be taken as a baseline LoS probability model for comparison with the proposed one in this work.

It is clear to mention that the proposed model in (29) tends to 1 by scaling $\theta=\arctan \left(\Delta h_{\mathrm{t}, \mathrm{r}} / D\right) \rightarrow \pi / 2$ (i.e., aerial platform stands right atop the ground $\mathrm{Rx}$ ), which comes by shrinking the Tx-Rx horizontal distance (i.e., $D \rightarrow 0$ ) or by having a sufficiently high altitude of the aerial platform (i.e., $\left.h_{\mathrm{t}} \rightarrow \infty\right)$. Alternatively, LoS probability can shrink to almost 0 as $\theta \rightarrow 0$, which can be achieved by scaling $D \rightarrow \infty$, while the scenario of $\Delta h_{\mathrm{t}, \mathrm{r}} \rightarrow 0$ is not feasible when assuming AtG channels. However, in the context of air-to-air (AtA) channels, Tx and $\mathrm{Rx}$ can operate at approximately comparable altitudes (i.e., $\Delta h_{\mathrm{t}, \mathrm{r}} \rightarrow 0$ ), which gives $\theta \rightarrow 0$ but does not necessarily result in a very small LoS probability as in the case of AtG channels. In such a context, (26) reduces to

$$
z_{k}=h_{\mathrm{r}}-b_{\eta} \sqrt{1-\frac{\left(\frac{\ell}{2}-y_{k}\right)^{2}}{a^{2}}} .
$$

To capture the scenario where $h_{\mathrm{t}} \simeq h_{\mathrm{r}}$, we suggest to revisit (29) as

$$
\mathrm{P}_{\mathrm{LoS}}(D)=1-\exp \left(-m_{\mathrm{f}}\left(\overline{\frac{\Delta h_{\mathrm{t}, \mathrm{r}}}{D}}\right)^{n_{\mathrm{f}}}\right),
$$

where $\overline{\Delta h_{\mathrm{t}, \mathrm{r}}}=\delta_{\mathrm{tr}} \bar{h}+\left(1-\delta_{\mathrm{tr}}\right) \Delta h_{\mathrm{t}, \mathrm{r}}$ such that $\bar{h}=\left(h_{\mathrm{r}}+h_{\mathrm{t}}\right) / 2$ and $\delta_{\text {tr }}$ is the Kronecker delta defined as

$$
\delta_{\mathrm{tr}}= \begin{cases}1 & \text { if } h_{\mathrm{t}} \simeq h_{\mathrm{r}} \\ 0 & \text { if } h_{\mathrm{t}} \gg h_{\mathrm{r}} .\end{cases}
$$

Fig. 7 illustrates the differences between the proposed LoS probability model in (29) that fit the calculated data from (25) based on (26) and the simple modified Sigmoid function presented in [13] that fit the calculated data from (25) based on (27). By analyzing such differences, one can observe that the deviation from the direct line based model becomes tight as the transmission frequency increases. This is due actually to the observation that the semi-minor axis of the 1st Fresnel ellipse is frequency-dependent where $b \rightarrow 0$ as $f \rightarrow \infty$ and then (26)

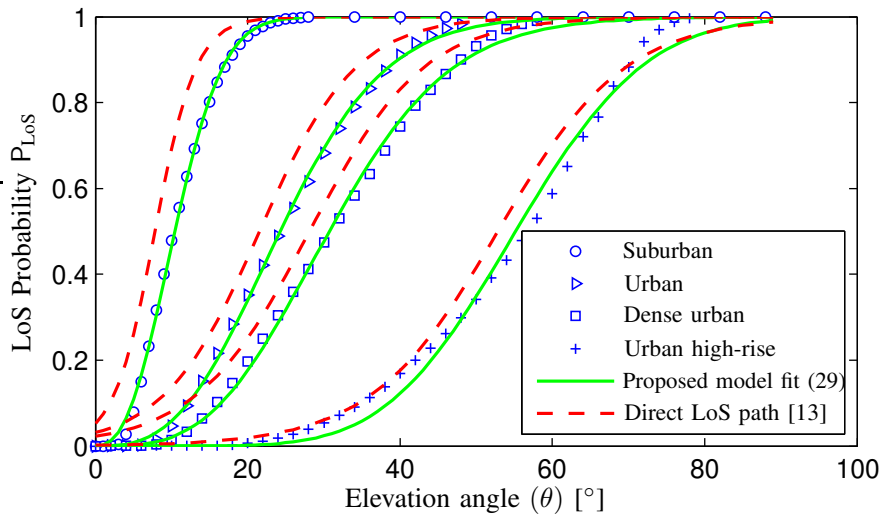

(a)

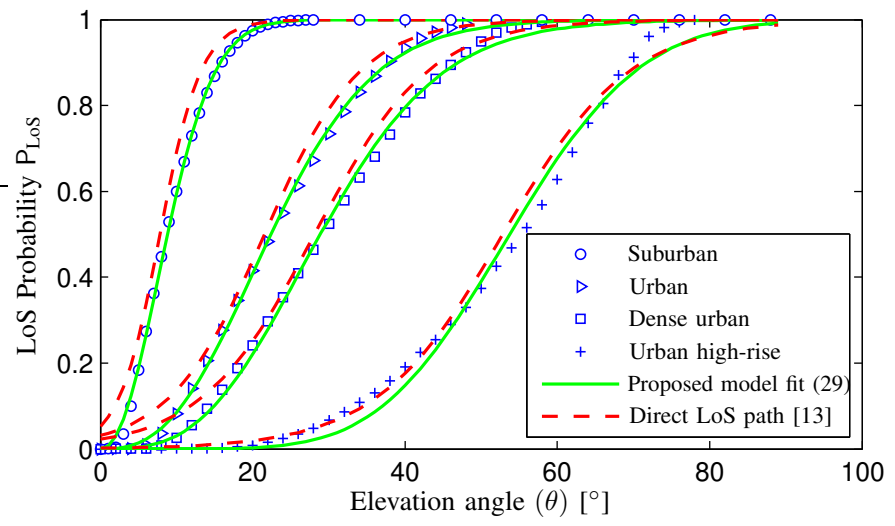

(b)

Fig. 7. The proposed LoS probability model fitting calculated data from (25) based on (26) in different propagation environments and the simple modified Sigmoid function presented in [13] that fit the calculated data from (25) based on (27). Parameters: $h_{\mathrm{r}}=2 \mathrm{~m}, h_{\mathrm{t}}=5 \mathrm{~km}$, and (a) $f=700 \mathrm{MHz}$ and (b) $f=3.5 \mathrm{GHz}$.

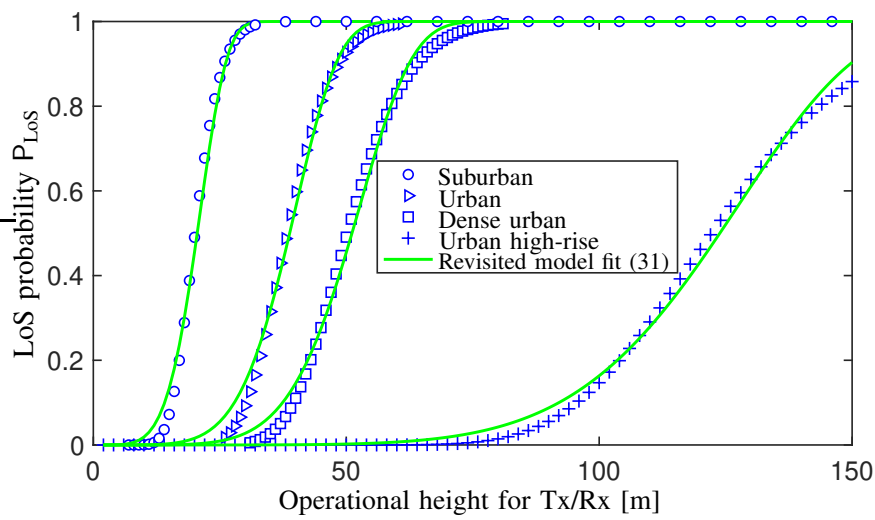

Fig. 8. The proposed LoS probability model as a function of Rx/Tx height (i.e., $h_{\mathrm{t}} \simeq h_{\mathrm{r}}$ ) in different propagation environments for $D=1000 \mathrm{~m}$ and $f=3.5 \mathrm{GHz}$.

reduces to (27). Furthermore, Fig. 8 shows how the revisited LoS probability model in (31) - to capture the scenario where Tx and Rx have comparable altitudes in an AtA fashion (i.e., $h_{\mathrm{t}} \simeq h_{\mathrm{r}}$ ) - can fit the calculated data from (25) based on (30).

In Table III, we estimate the empirical parameters $n_{\mathrm{f}}$ and $m_{\mathrm{f}}$ of the proposed model in (29) from the data in (25) calculated using (26) for different transmission frequencies and Tx and $\mathrm{Rx}$ altitudes in such a way to minimize the RMS deviation. In this way, by taking $\mathrm{RMS}_{\mathrm{f}}$ obtained from the deviation between the model in (29) and the data calculated using (26) and 
TABLE III

EMPIRICAL PARAMETERS OF LOS PROBABILITY MODELS BASED ON THE DIRECT LINK AND $60 \%$ OF THE 1 ST FRESNEL ZONE ASSOCIATED WITH RMS CALCULATIONS. (a) $h_{r}=2 \mathrm{~m}$ and (b) $h_{r}=20 \mathrm{~m}$.

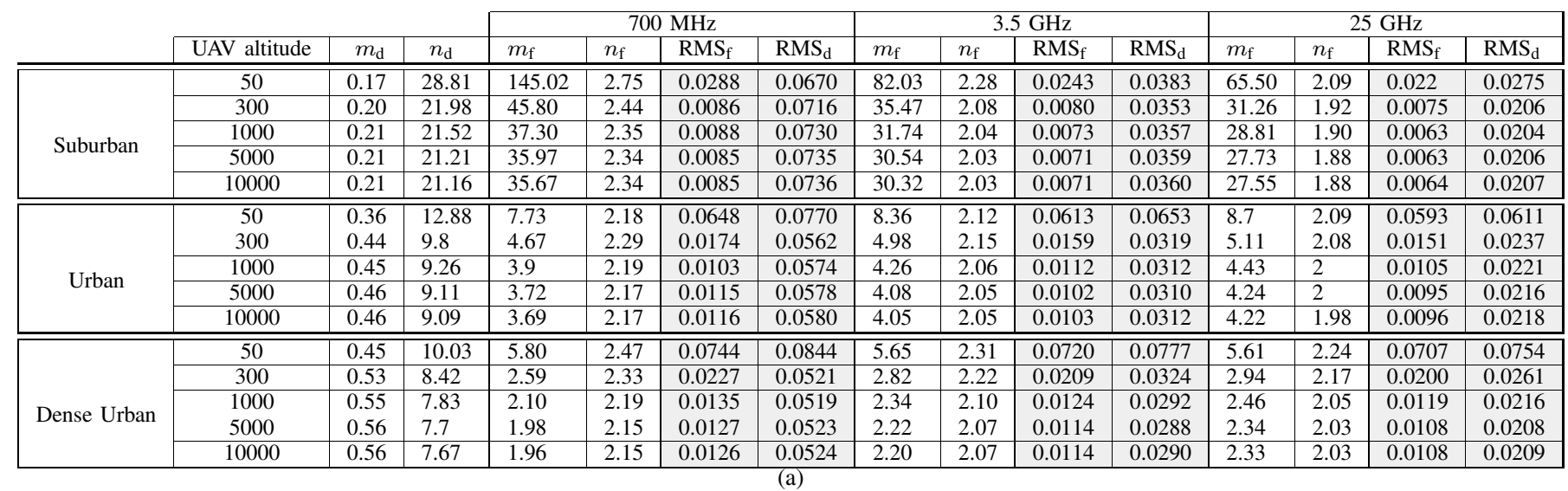

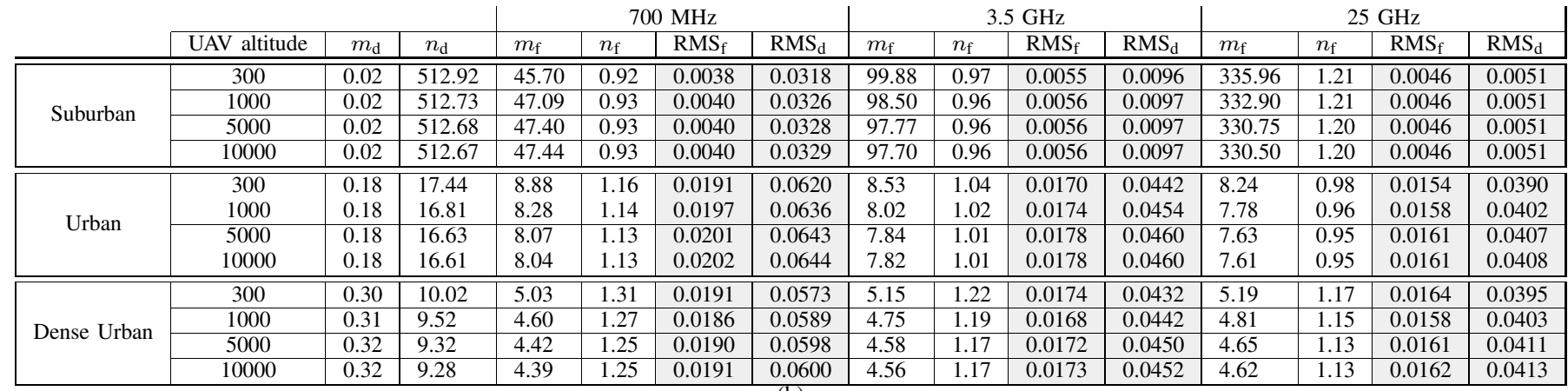

(b)

comparing it with $\mathrm{RMS}_{\mathrm{d}}$ obtained from the deviation between the popular model developed in [13] and the data calculated using (27), one can observe that the RMS is greatly enhanced, particularly when assuming those scenarios where the impact of randomness in the blockages height is diminished (i.e., considering for instance sufficiently high UAV altitudes and/or propagation environments such as suburban, urban, and even dense urban). In particular, the gap between the calculated data of the LoS probability and the direct line based model shrinks with increasing frequency due to the dependence of the semi-minor axis $b$ of the 1st Fresnel ellipse on frequency. This observation can be verified both visually from Fig. 7 and numerically from Table III where $\mathrm{RMS}_{\mathrm{d}}$ decreases with increasing frequency. In addition, for a given urban environment and frequency, Table III shows that the empirical parameters $m_{\mathrm{f}}$ and $n_{\mathrm{f}}$ become almost independent from the UAV altitude for higher altitude values (i.e., $h_{\mathrm{t}}>1000 \mathrm{~m}$ ). In such a context, performance evaluation can be performed with no need to repeatedly calculate the empirical parameters at each given altitude.

Besides its accuracy improvements, the proposed model in (29) has more tractability allowing to develop closed-form expressions of some measures of interest as will be discussed in the following section. This is notably granted since the following integral based on (29) can be derived in closed-form as [30, Eq. 2.325.6]

$$
\Lambda(x)=\int_{0}^{u} x\left(1-\mathrm{P}_{\mathrm{LoS}}(x)\right) \mathrm{d} x
$$

$$
=\frac{{\overline{\Delta h_{\mathrm{t}, \mathrm{r}}}}^{2}\left(m_{\mathrm{f}}\right)^{\frac{2}{n_{\mathrm{f}}}}}{n_{\mathrm{f}}} \Gamma\left(-\frac{2}{n_{\mathrm{f}}}, m_{\mathrm{f}}\left(\frac{\overline{\Delta h_{\mathrm{t}, \mathrm{r}}}}{u}\right)^{n_{\mathrm{f}}}\right) .
$$

Nevertheless, $\Lambda(\cdot)$ under the LoS models proposed in [13], [18] remains unsimplified in view of their construction as a composite of the functions $\exp (),. \arctan ($.$) , and 1 / x$, which may require efficient numerical methods to evaluate $\Lambda(\cdot)$ and hence increases computational complexity when used in extended PDF formulations.

\section{Modeling And Analysis of Coverage with AERIAL PlatForms}

In this section, we will take advantage of the LoS probability developed earlier in (29) to evaluate the downlink performance of a UE served by an aerial platform. We will consider i) the case of a fixed aerial platform location, and ii) the case where aerial platforms are randomly distributed according to a PPP around the typical ground UE.

Propagation through the wireless channel experiences both fading and path loss. Let $g$ represent the channel power fading gain between Tx and Rx. Specifically, we assume that the channel amplitude for LoS propagation conditions are Nakagami- $m$ distributed, i.e., $g_{\text {LoS }} \sim \operatorname{Gamma}(m, 1 / m)$, and the channel amplitude for NLoS propagation are Rayleigh distributed, i.e., $g_{\mathrm{NLOS}} \sim \exp (1)$. In addition, we adopt the standard power-law pathloss model such that, depending on the propagation group (i.e., LoS or NLoS connection) between 
UE and aerial platform, the RSS at the UE of interest is given by [13]

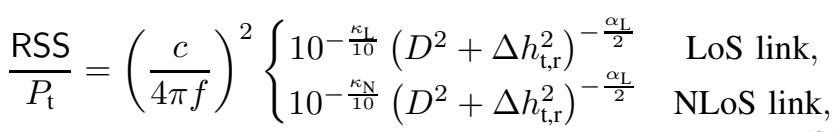

where $\alpha_{\mathrm{L}}$ is the PLE over the channel for LoS propagation and $\kappa_{\mathrm{L}}$ and $\kappa_{\mathrm{N}}$ are the mean value of the excessive pathloss in $\mathrm{dB}$ due to the LoS and NLoS connections, respectively. As in [13], the following typical pairs $(0.1,21),(1.0,20),(1.6,23)$, and $(2.3,34)$ will be used for $\left(\kappa_{\mathrm{L}}, \kappa_{\mathrm{N}}\right)$ in suburban, urban, dense urban, and high-rise urban environments, respectively. For convenience, we consider the following shorthands: $K=$ $10^{\frac{\kappa_{\mathrm{N}}-\kappa_{\mathrm{L}}}{5 \alpha_{\mathrm{L}}}}, \omega_{\mathrm{L}}=10^{\frac{\kappa_{\mathrm{L}}}{10}}\left(\frac{4 \pi f}{c}\right)^{2}$, and $\omega_{\mathrm{N}}=10^{\frac{\kappa_{\mathrm{N}}}{10}}\left(\frac{4 \pi f}{c}\right)^{2}$.

We consider $\sigma^{2}=W N_{0}$ as the noise power expressed as a function of the transmission bandwidth $W$ and noise power density $N_{0}=10^{\frac{N_{0}(\mathrm{dBm})}{10}}$ with $N_{0}(\mathrm{dBm})=-174+\mathcal{F}_{\mathrm{dB}}$ and $\mathcal{F}_{\mathrm{dB}}$ being the noise figure in $\mathrm{dB}$ assumed to be constant with respect to the operating frequency.

Unless stated otherwise, we consider the following parameters in the numerical analysis: a standard PLE $\alpha_{\mathrm{L}}=2$, the Fresnel critical clearance zone is parametrized by $\eta=0.6$, a UE height $h_{\mathrm{r}}=2 \mathrm{~m}, f=3.5 \mathrm{GHz}, W=25 \mathrm{MHz}$, and a shape parameter $m=10$ (which corresponds to a Rician K-factor of $13 \mathrm{~dB}$ [17]).

\section{A. A Priori Known Location of the Aerial Platform}

When the horizontal distance $D$ between the UE of interest and the aerial platform is known a priori (i.e., deterministic), the coverage probability of the UE is defined as the probability $\mathcal{P}_{\text {cov }}$ of receiving a downlink SNR greater than a given threshold $T$ from the aerial platform. In other words, coverage probability here can be seen as the fraction of the disk of radius $D$ that is covered with an SNR greater than $T$. In this way, $\mathcal{P}_{\text {cov }}$ can be derived as

$$
\begin{aligned}
& \mathcal{P}_{\text {cov }}=\mathbb{P}\left(g_{\mathrm{LoS}} \frac{\mathrm{RSS}}{\sigma^{2}}>T, \operatorname{LoS} \text { Link }\right) \\
& \quad+\mathbb{P}\left(g_{\mathrm{NLoS}} \frac{\mathrm{RSS}}{\sigma^{2}}>T, \text { NLoS Link }\right) \\
& \stackrel{(a)}{=} \mathbb{P}\left(g_{\mathrm{LoS}}>\frac{\omega_{\mathrm{L}} \sigma^{2} T}{P_{t}}\left(D^{2}+\Delta h_{\mathrm{t}, \mathrm{r}}^{2}\right)^{\frac{\alpha_{\mathrm{L}}}{2}}\right) \mathrm{P}_{\mathrm{LoS}}(D) \\
& +\mathbb{P}\left(g_{\mathrm{NLoS}}>\frac{\omega_{\mathrm{N}} \sigma^{2} T}{P_{t}}\left(D^{2}+\Delta h_{\mathrm{t}, \mathrm{r}}^{2}\right)^{\frac{\alpha_{\mathrm{L}}}{2}}\right)\left(1-\mathrm{P}_{\mathrm{LoS}}(D)\right) \\
& \stackrel{(b)}{=} \frac{\Gamma\left(m, \frac{m \omega_{\mathrm{L}} \sigma^{2} T}{P_{t}}\left(D^{2}+\Delta h_{\mathrm{t}, \mathrm{r}}^{2}\right)^{\frac{\alpha_{\mathrm{L}}}{2}}\right)}{\Gamma(m)}\left(1-\exp \left(-m_{\mathrm{f}}\left(\frac{\Delta h_{\mathrm{t}, \mathrm{r}}}{D}\right)^{n_{\mathrm{f}}}\right)\right) \\
& +\exp \left(-\frac{\omega_{\mathrm{N}} \sigma^{2} T}{P_{t}}\left(D^{2}+\Delta h_{\mathrm{t}, \mathrm{r}}^{2}\right)^{\frac{\alpha_{\mathrm{L}}}{2}}-m_{\mathrm{f}}\left(\frac{\Delta h_{\mathrm{t}, \mathrm{r}}}{D}\right)^{n_{\mathrm{f}}}\right),
\end{aligned}
$$

where (a) results from splitting RSS according the LoS and NLoS propagation groups and (b) follows by calculating the complementary CDF of the channel amplitude for LoS and NLoS propagation conditions, respectively.

Fig. 9 presents the trend of coverage probability when considering the probability of $\operatorname{LoS}$ in (29) with empirical

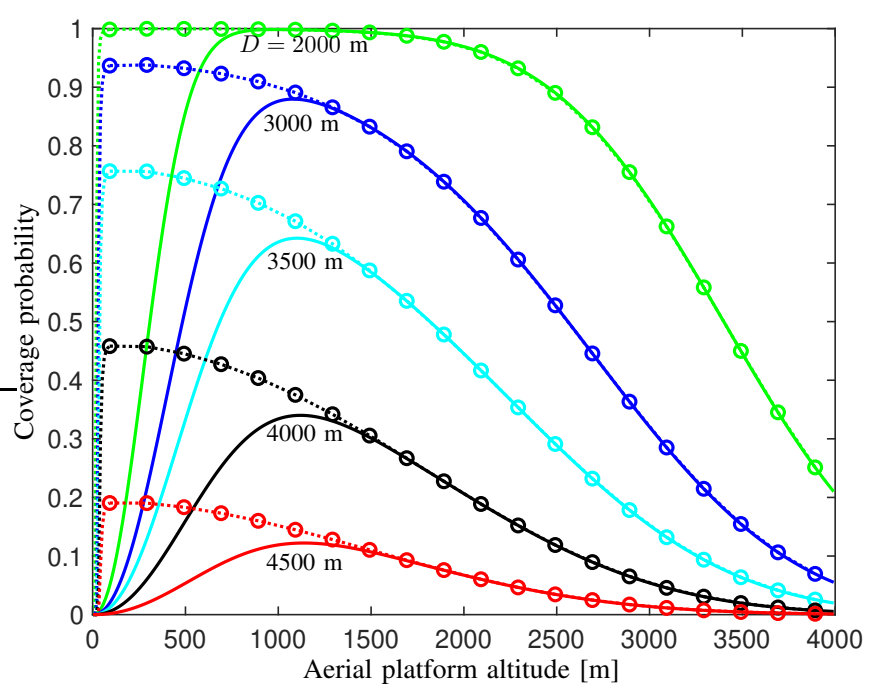

Fig. 9. Coverage probability as a function of the aerial platform altitude and horizontal distance $D$ for $T=10 \mathrm{~dB}$ and $P_{\mathrm{t}}=37 \mathrm{dBm}$ in high-rise urban environment. The solid lines represent the proposed $\mathrm{P}_{\mathrm{LoS}}$ model with empirical parameters fitted with fixed altitude $1000 \mathrm{~m}$, lines with markers represent the calculated $\mathrm{P}_{\mathrm{LoS}}^{\mathrm{c}}$ from (26) as a function of the aerial platform altitude.

parameters computed at a fixed altitude $h_{\mathrm{t}}=1000 \mathrm{~m}$ (solid line), and the probability of LoS calculated from (26) for several altitudes of the aerial platform (circle line). It is obvious that both models are in agreement for high values of the aerial platform altitude $\left(h_{\mathrm{t}}>1000 \mathrm{~m}\right)$ at which the empirical parameters $m_{\mathrm{f}}$ and $n_{\mathrm{f}}$ of the model in (29) become weakly dependent on altitude $h_{\mathrm{t}}$ and hence no need to recalculate them for every altitude value (see Table III). Further, the two data-set strands in Fig. 9 (computed and analytical) support the observation that the coverage probability function is unimodal, which yields an optimal altitude range that maximizes the coverage probability. These optimal values are in fact the result of a tradeoff between the likelihood of a link being in LoS and the path loss, both growing with altitude, which ultimately can lead to the joint optimization of communication and energy consumption of aerial platforms, and many other parameters and resources.

\section{B. Random Location of the Aerial Platform}

Here, the horizontal distance $D$ between the UE of interest and its serving aerial platform is considered to be a random variable. In such a context, we consider a cellular network comprised of aerial platforms elevated at an average altitude $h_{\mathrm{t}}$ with locations modeled by a $2 \mathrm{D}$ homogeneous PPP $\Psi_{\mathrm{t}}$ with density $\lambda_{\mathrm{t}}$ [number of aerial platforms $\left./ \mathrm{m}^{2}\right]$. Without loss of generality and as permitted by the Slivnyak-Mecke's theorem [29, Th. 1.4.5], the typical UE, denoted by $\mathrm{UE}_{0}$ and with altitude $h_{\mathrm{r}}$, is located at the origin and taken as the object of the analysis.

The coverage probability of $\mathrm{UE}_{0}$ is defined as the probability $\mathcal{P}_{\text {cov }}$ of receiving a downlink SNR greater than a certain threshold $T$ from the best aerial platform in terms of the lowest 


$$
\begin{aligned}
f_{D}^{\mathrm{L}}(u)= & 2 \pi \lambda_{\mathrm{t}} u \exp \left(-\pi \lambda_{\mathrm{t}} u^{2}\right)\left(1-\exp \left(-m_{\mathrm{f}}\left(\frac{\Delta h_{\mathrm{t}, \mathrm{r}}}{u}\right)^{n_{\mathrm{f}}}\right)\right) \exp \left(2 \pi \lambda_{\mathrm{t}} \frac{\Delta h_{\mathrm{t}, \mathrm{r}}^{2}\left(m_{\mathrm{f}}\right)^{\frac{2}{n_{\mathrm{f}}}}}{n_{\mathrm{f}}} \Gamma\left(-\frac{2}{n_{\mathrm{f}}}, m_{\mathrm{f}}\left(\frac{\Delta h_{\mathrm{t}, \mathrm{r}}}{u}\right)^{n_{\mathrm{f}}}\right)\right) \\
& \times \exp \left(-2 \pi \lambda_{\mathrm{t}} \frac{\Delta h_{\mathrm{t}, \mathrm{r}}^{2}\left(m_{\mathrm{f}}\right)^{\frac{2}{n_{\mathrm{f}}}}}{n_{\mathrm{f}}} \Gamma\left(-\frac{2}{n_{\mathrm{f}}}, m_{\mathrm{f}}\left(\frac{\Delta h_{\mathrm{t}, \mathrm{r}}}{\psi(u)}\right)^{n_{\mathrm{f}}}\right)\right), \\
f_{D}^{\mathrm{N}}(u)= & 2 \pi \lambda_{\mathrm{t}} u \exp \left(-\pi \lambda_{\mathrm{t}}(\varrho(u))^{2}\right) \exp \left(-m_{\mathrm{f}}\left(\frac{\Delta h_{\mathrm{t}, \mathrm{r}}}{u}\right)^{n_{\mathrm{f}}}\right) \exp \left(-2 \pi \lambda_{\mathrm{t}} \frac{\Delta h_{\mathrm{t}, \mathrm{r}}^{2}\left(m_{\mathrm{f}}\right)^{\frac{2}{n_{\mathrm{f}}}}}{n_{\mathrm{f}}} \Gamma\left(-\frac{2}{n_{\mathrm{f}}}, m_{\mathrm{f}}\left(\frac{\Delta h_{\mathrm{t}, \mathrm{r}}}{u}\right)^{n_{\mathrm{f}}}\right)\right) \\
& \times \exp \left(2 \pi \lambda_{\mathrm{t}} \frac{\Delta h_{\mathrm{t}, \mathrm{r}}^{2}\left(m_{\mathrm{f}}\right)^{\frac{2}{n_{\mathrm{f}}}}}{n_{\mathrm{f}}} \Gamma\left(-\frac{2}{n_{\mathrm{f}}}, m_{\mathrm{f}}\left(\frac{\Delta h_{\mathrm{t}, \mathrm{r}}}{\varrho(u)}\right)^{n_{\mathrm{f}}}\right)\right),
\end{aligned}
$$

where $\psi(u)=\sqrt{\max \left(\frac{u^{2}+\Delta h_{\mathrm{t}, \mathrm{r}}^{2}}{K}-\Delta h_{\mathrm{t}, \mathrm{r}}^{2}, 0\right)}$ and $\varrho(u)=\sqrt{K u^{2}+(K-1) \Delta h_{\mathrm{t}, \mathrm{r}}^{2}}$.

path loss criterion [22]. Formally,

$$
\begin{aligned}
& \mathcal{P}_{\text {cov }}=\mathbb{P}\left(g_{\mathrm{LoS}} \frac{\mathrm{RSS}}{\sigma^{2}}>T, \text { LoS Link }\right) \\
& +\mathbb{P}\left(g_{\mathrm{NLoS}} \frac{\mathrm{RSS}}{\sigma^{2}}>T, \text { NLoS Link }\right) \\
& \stackrel{(a)}{=} \int_{0}^{\infty} \mathbb{P}\left(g_{\mathrm{LoS}}>\frac{\omega_{\mathrm{L}} \sigma^{2} T}{P_{t}}\left(u^{2}+\Delta h_{\mathrm{t}, \mathrm{r}}^{2}\right)^{\frac{\alpha_{\mathrm{L}}}{2}}\right) f_{D}^{\mathrm{L}}(u) \mathrm{d} u \\
& +\int_{0}^{\infty} \mathbb{P}\left(g_{\mathrm{NLoS}}>\frac{\omega_{\mathrm{N}} \sigma^{2} T}{P_{t}}\left(u^{2}+\Delta h_{\mathrm{t}, \mathrm{r}}^{2}\right)^{\frac{\alpha_{\mathrm{L}}}{2}}\right) f_{D}^{\mathrm{N}}(u) \mathrm{d} u \\
& \stackrel{(b)}{=} \int_{0}^{\infty} \frac{\Gamma\left(m, \frac{m \omega_{\mathrm{L}} \sigma^{2} T}{P_{t}}\left(u^{2}+\Delta h_{\mathrm{t}, \mathrm{r}}^{2}\right)^{\frac{\alpha_{\mathrm{L}}^{\mathrm{L}}}{2}}\right)}{\Gamma(m)} f_{D}^{\mathrm{L}}(u) \mathrm{d} u \\
& +\int_{0}^{\infty} \exp \left(-\frac{\omega_{\mathrm{N}} \sigma^{2} T}{P_{t}}\left(u^{2}+\Delta h_{\mathrm{t}, \mathrm{r}}^{2}\right)^{\frac{\alpha_{\mathrm{L}}}{2}}\right) f_{D}^{\mathrm{N}}(u) \mathrm{d} u,
\end{aligned}
$$

where $f_{D}^{\mathrm{L}}$ and $f_{D}^{\mathrm{N}}$ in (a) and (b) are the PDFs of the horizontal distance between $\mathrm{UE}_{0}$ and the best aerial platform from $\Psi_{\mathrm{t}}$ in terms of the lowest path loss association rule, in LoS and NLoS propagations, respectively.

In fact, the horizontal distance from $\mathrm{UE}_{0}$ to the nearest aerial platform of $\Psi_{\mathrm{t}}$ among the ones with LoS and NLoS propagations are denoted by $D^{\mathrm{L}}$ and $D^{\mathrm{N}}$, respectively. Their CDFs are obtained from the null probability of the PPP as

$$
\begin{gathered}
F_{D^{\mathrm{L}}}(u)=1-\exp \left(-2 \pi \lambda_{\mathrm{t}} \int_{0}^{u} x \mathrm{P}_{\mathrm{LoS}}(x) \mathrm{d} x\right), \\
F_{D^{\mathrm{N}}}(u)=1-\exp \left(-2 \pi \lambda_{\mathrm{t}} \int_{0}^{u} x\left(1-\mathrm{P}_{\mathrm{LoS}}(x)\right) \mathrm{d} x\right) .
\end{gathered}
$$

The CDF of the horizontal distance $D$ between $\mathrm{UE}_{0}$ and the best aerial platform of $\Psi_{\mathrm{t}}$ in terms of the lowest path loss association rule, in LoS propagations is derived as

$$
\begin{aligned}
& F_{D}^{\mathrm{L}}(u)=\mathbb{P}\left(D^{\mathrm{L}}<u,\left(D^{\mathrm{N}}\right)^{2}>\frac{\left(D^{\mathrm{L}}\right)^{2}+\Delta h_{\mathrm{t}, \mathrm{r}}^{2}}{K}-\Delta h_{\mathrm{t}, \mathrm{r}}^{2}\right) \\
& \left.=\int_{0}^{u}\left(1-F_{D^{\mathrm{N}}}\left(\sqrt{\max \left(\frac{u^{2}+\Delta h_{\mathrm{t}, \mathrm{r}}^{2}}{K}-\Delta h_{\mathrm{t}, \mathrm{r}}^{2}, 0\right)}\right)\right)\right) \mathrm{d} F_{D^{\mathrm{L}}}(x) .
\end{aligned}
$$

The exact expression of the PDF in (36) is then obtained by deriving (35) with respect to $u$ and using (33) for further

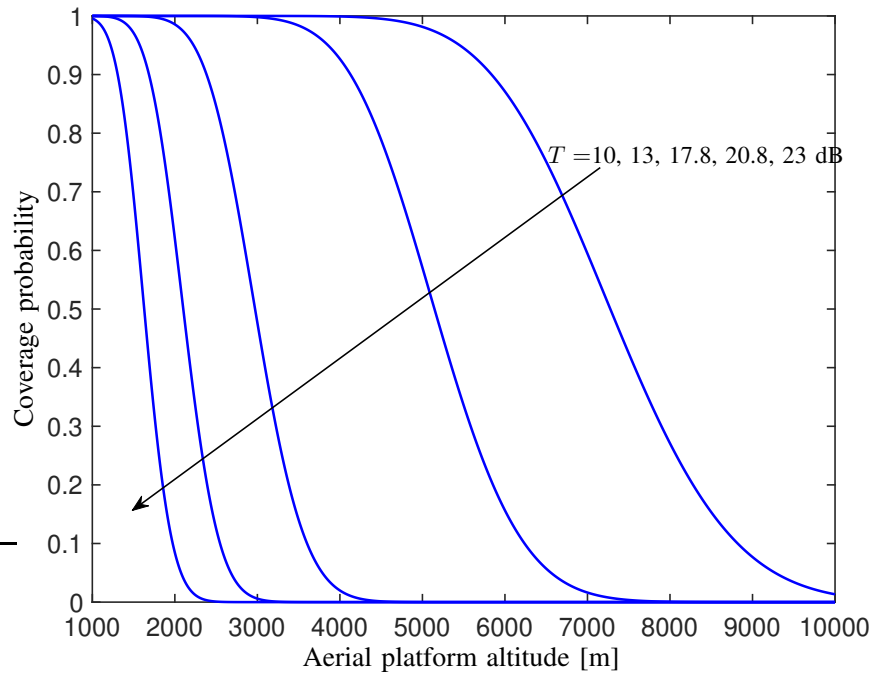

Fig. 10. Coverage probability as a function of the aerial platform altitude and the SNR target $T$ for $P_{\mathrm{t}}=43 \mathrm{dBm}$.

simplifications. A similar approach is followed to derive the PDF of the NLoS case in (37).

The tractability of the proposed LoS probability model in (29) has particularly permitted to elaborate a closed-form expression of $f_{D}^{\mathrm{L}}$ and $f_{D}^{\mathrm{N}}$ - the PDFs of the horizontal distance between $\mathrm{UE}_{0}$ and the best aerial platform from $\Psi_{\mathrm{t}}$ in terms of the lowest path loss association rule, in LoS and NLoS propagations, respectively - which increases the computational efficiency of coverage probability in (32) as compared to other widely used models in the literature [13].

Fig. 10 illustrates the average coverage probability over the horizontal distance between $\mathrm{UE}_{0}$ and its serving aerial platform as a function of altitude and SNR target $T$. In particular, we can observe that the coverage probability is a monotonically decreasing function with $h_{\mathrm{t}}$ and $T$ for sufficiently higher altitudes. For a given minimum required coverage probability $\mathcal{P}_{\text {cov }}^{0}$, the aerial platform altitude can be increased to a particular height $h_{\mathrm{t}}^{0}$ to keep that coverage probability requirement, beyond this altitude $h_{\mathrm{t}}^{0}$ it is inevitable to reduce the SNR threshold $T$ to maintain the desired coverage probability requirement. 


\section{CONCLUSION}

In this work, we evaluated a key component of the AtG channels in the emerging 5G/B5G cellular networks being deployed on several specific sub-6GHz and mmWave frequency bands as projected by 3GPP-R15 and IMT-2020 requirements. It consists of a LoS probability model that, as opposed to the widely adopted models developed in the literature, is both accurate and mathematically tractable. It takes into account the scattering mechanisms such as reflection and diffraction that suggest, as we have shown through the two-ray propagation and KED models, that the environmental obstacles should not intrude into $60 \%$ of the 1 st Fresnel ellipsoid between Tx and $\mathrm{Rx}$ to ensure communication under FSPL (i.e., PLE $\simeq 2$ ). In addition, it enables, under the abstraction of a powerful analytical tool like stochastic geometry, the development of closed-form expression for PDFs of interest, which facilitates a seamless and physically meaningful system-level performance analysis of future $5 \mathrm{G} / \mathrm{B} 5 \mathrm{G}$ networks in the presence of airborne platforms.

For future extensions of this work, it would be interesting to explore the merits of the proposed model in terms of accuracy and analytical flexibility to address the joint optimization of communication and other relevant resources such as the energy efficiency of ground and/or airborne networks, data delivery delay by aerial platforms from data aggregators in the context of the IoT ecosystem, and the joint access and backhaul communication via aerial platforms.

\section{ACKNOWLEDGMENTS}

This work was partially funded by Institut Carnot Télécom \& Société Numérique under project Robot4IoT and by Pôle de Recherche Avancée en Communications (PRACOM), France.

\section{REFERENCES}

[1] ITU-R, "Technical feasibility of IMT in bands above $6 \mathrm{GHz}$," International Telecommunication Union (ITU), M Series ITU-R M.2376-0, Jul. 2015.

[2] I. Bor-Yaliniz and H. Yanikomeroglu, "The new frontier in RAN heterogeneity: Multi-tier drone-cells,"IEEE Commun. Mag., vol. 54, no. 11, pp. 48-55, Nov. 2016.

[3] 3GPP, "Study on New Radio (NR) to support non terrestrial networks (Release 15)," 3rd Generation Partnership Project, technical specification group radio access network 3GPP TR 38.811 V15.0.0, Aug. 2018.

[4] 3GPP, "Enhancement for unmanned aerial vehicles," 3rd Generation Partnership Project, technical specification group services and system aspects, 3GPP TR 22.829 V17.1.0, Sep. 2019.

[5] 3GPP, "Unmanned aerial system (UAS) support in 3GPP," 3rd Generation Partnership Project, technical specification group services and system aspects, 3GPP TS 22.125 V17.2.0, Sep. 2020.

[6] S. Alfattani, W. Jaafar, Y. Hmamouche, H. Yanikomeroglu, A. Yongaçoglu, N. D. Dào, and P. Zhu, "Aerial platforms with reconfigurable smart surfaces for 5G and beyond," IEEE Commun. Mag., vol. 59, no. 1, pp. 96-102, Jan. 2021.

[7] W. Khawaja, I. Guvenc, D. W. Matolak, U. -C. Fiebig, and N. Schneckenburger, "A survey of air-to-ground propagation channel modeling for unmanned aerial vehicles," IEEE Commun. Surveys Tuts, vol. 21, no. 3, pp. 2361-2391, Thirdquarter 2019.
[8] M. K. Samimi, T. S. Rappaport, and G. R. MacCartney, "Probabilistic omnidirectional path loss models for millimeter-wave outdoor communications,'IEEE Wireless Commun. Lett., vol. 4, no. 4, pp. 357-360, 2015.

[9] 3GPP, "Study on channel model for frequencies from 0.5 to $100 \mathrm{GHz}$ (Rel. 14)," 3rd Generation Partnership Project, technical specification group radio access network, 3GPP TR 38.901 V1.0.1, Mar. 2017.

[10] ITU-R, "Guidelines for evaluation of radio interface technologies for imt2020," International Telecommunication Union (ITU), M Series ITUR M.2412-0, Oct.2017.

[11] 3GPP, "Study on enhanced LTE support for aerial vehicles," 3rd Generation Partnership Project, technical specification group radio access network, 3GPP TR 36.777 V1.0.0, Dec. 2017.

[12] D. He, B. Ai, K. Guan, L. Wang, Z. Zhong, and T. Kürner, "The design and applications of high-performance ray-tracing simulation platform for 5G and beyond wireless communications: A tutorial," IEEE Commun. Surveys Tuts, vol. 21, no. 1, pp. 10-27, Firstquarter 2019.

[13] A. Hourani, K. Sithamparanathan, and S. Lardner, "Optimal LAP altitude for maximum coverage," IEEE Wireless Commun. Lett., vol. 3, no. 6, pp. 569-572, Dec. 2014.

[14] T. Bai, R. Vaze, and R. W. Heath, "Analysis of blockage effects on urban cellular networks," IEEE Trans. Wireless Commun., vol. 13, no. 9, pp. 5070-5083, Sep. 2014.

[15] A. Al-Hourani, "On the probability of line-of-sight in urban environments," IEEE Wireless Commun. Lett., vol. 9, no. 8, pp. 1178-1181, Aug. 2020.

[16] M. Gapeyenko, D. Moltchanov, S. Andreev, and R. W. Heath, "Line-ofsight probability for mmWave-based UAV communications in 3D urban grid deployments," IEEE Trans. Wireless Commun., to appear.

[17] I. Atzeni, J. Arnau, and M. Kountouris, "Downlink cellular network analysis with LOS/NLOS propagation and elevated base stations," IEEE Trans. Wireless Commun., vol. 17, no. 1, pp. 142-156, Jan. 2018.

[18] J. Holis and P. Pechac, "Elevation dependent shadowing model for mobile communications via high altitude platforms in built-Up areas," IEEE Trans. Antennas and Propag., vol. 56, no. 4, pp. 1078-1084, April 2008.

[19] L. Zhou, Z. Yang, G. Zhao, S. Zhou, and C. Wang, "Propagation characteristics of air-to-air channels in urban environments," in Proc. IEEE Global Commun. Conf. (GLOBECOM), Abu Dhabi, UAE, Dec. 2018, pp. $1-6$

[20] X. Liu, J. Xu, and H. Tang, "Analysis of frequency-dependent line-ofsight probability in 3-D environment," IEEE Commun. Lett., vol. 22, no. 8, pp. 1732-1735, Aug. 2018.

[21] Z. Cui, K. Guan, C. B.-Rodríguez, B. Ai, and Z. Zhong, "Frequencydependent line-of-sight probability modeling in built-up environments," IEEE Internet Things J., vol. 7, no. 1, pp. 699-709, Jan. 2020.

[22] Y. Hmamouche, M. Benjillali, S. Saoudi, H. Yanikomeroglu, and M. D. Renzo, "New trends in stochastic geometry for wireless networks: A tutorial and survey," Proc. IEEE, vol. 109, no. 7, pp. 1200-1252, Jul. 2021.

[23] H. S. Dhillon and J. G. Andrews, "Downlink rate distribution in heterogeneous cellular networks under generalized cell selection," IEEE Wireless Commun. Lett., vol. 3, no. 1, pp. 42-45, Feb. 2014.

[24] ITU-R,"Propagation data and prediction methods required for the design of terrestrial broadband radio access systems operating in a frequency range from 3 to $60 \mathrm{GHz}$," International Telecommunication Union (ITU), P Series ITU-R P.1410-5, Feb.2012.

[25] T. S. Rappaport, Wireless Communications, Principles and Practice, 2nd ed., Ed. USA: Prentice Hall, 2002.

[26] ITU-R,"Propagation by diffraction," International Telecommunication Union (ITU), P Series ITU-R P.526-15, Oct. 2019.

[27] A. AlAmmouri, J. G. Andrews, and F. Baccelli, "SINR and throughput of dense cellular networks with stretched exponential path loss," IEEE Trans. Wireless Commun., vol. 17, no. 2, pp. 1147-1160, Feb. 2018.

[28] H. S. Dhillon, R. K. Ganti, F. Baccelli, and J. G. Andrews, "Modeling and analysis of K-tier downlink heterogeneous cellular networks," IEEE J. Sel. Areas Commun., vol. 30, no. 3, pp. 550-560, Apr. 2012.

[29] F. Baccelli and B. Blaszczyszyn, Stochastic Geometry and Wireless Networks in Foundations and Trends in Networking, vol. 1, Now Publishers, 2009.

[30] I. S. Gradshteyn,I. M. Ryzhik, Table of Integrals, Series, and Products, 7th ed. Academic Press, Elsevier Inc, 2007. 


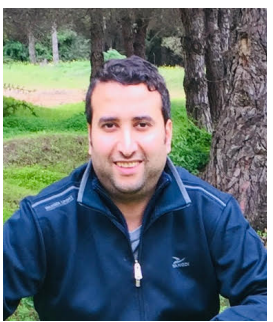

Yassine Hmamouche (M'20) received the Ph.D. degree in telecommunications from IMT Atlantique, Brest, France, in 2020.

Prior to joining academia, he extensively worked for eight years in the telecom industry as a radio network planning engineer and then as a project manager for several projects spanning different technologies. In 2019, he was a Visiting Researcher with Carleton University, Ottawa, ON, Canada. He is currently a Postdoctoral Fellow with IMT Atlantique. His current research interests include unmanned aerial vehicle (UAV) communication systems, reconfigurable intelligent surfaces, and Internet-of-Things (IoT) networks.

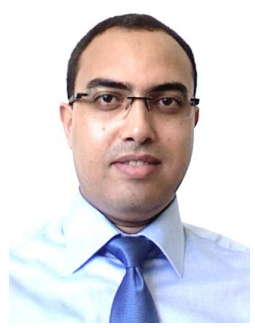

Mustapha Benjillali (SM'14) received the Ph.D. degree in telecommunications from Institut National de la Recherche Scientifique (INRS), Montreal, QC, Canada, in 2009.

He was a Post-doctoral Research Fellow with the Electrical Engineering Program, King Abdullah University of Science and Technology (KAUST), Saudi Arabia. He is currently a full professor with the Communications Systems Department, Institut National des Postes et Télécommunications (INPT),

Rabat, Morocco, where he is the Research Team Leader on Trends in Research on 5G-and-Beyond Technologies and EcoSystems (5TRonG). His current research interests are in the broad area of wireless communications for $6 \mathrm{G}$ applications, with a focus on mathematical and stochastic modeling, machine learning for communications, performance analysis, and optimal resource allocation strategies.

Dr. Benjillali serves on the editorial board of many leading international journals and in different organizational and TPC chairing roles in several major international IEEE conferences.

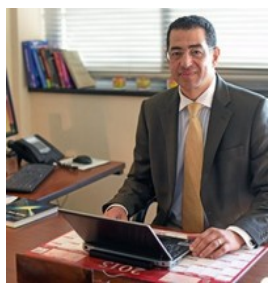

Samir Saoudi (SM'09) Samir SAOUDI was born in Rabat, Morocco, in 1963. He received the Electrical Engineering degree from École nationale supérieure des télécommunications de Bretagne (ENST-Bretagne), Brest, France, in 1987, the Ph.D degree in telecommunications from the Rennes I University, in 1990 (with France Telecom as industrial partner), and the "qualification to supervise research in science" degree in 1997.

Since 1991, he has been with Mathematical \& Electrical Engineering department at IMT Atlantique where he is currently a Full Professor. His research interests are speech and audio coding, non parametric probability density estimation, CDMA Techniques, Multi User Detection, MIMO systems, equalization and iterative processing with applications to mobile radio communications. His teaching interests are signal processing, probability, stochastic processes, speech processing, and Multi User Detection. During 1995-2005, he was head of the Master "Digital Communications Systems". He has supervised more than $35 \mathrm{PhD}$ Students. He has co-authored more than 30 journal papers and more than one hundred conference papers. During 2008-2015. He was head of Digital Communications Group of LabSticc (CNRS UMR N ${ }^{\circ} 6385$ ). Since April 2014, he has been head of Mathematical \& Electrical Engineering Department at IMT Atlantique (120 members).

Dr. Saoudi has been the general chair of the second International symposium on Image/Video Communications over fixed and mobile networks, IEEE ISIVC 2004. He has been the Co-Chair of the MIMO Systems Symposium and the IEEE International Wireless Communications and Mobile Computing Conference (IWCMC 2010), France. Since 2019, he has been the Steering Committee Chair of the IEEE ISIVC symposium. 